\title{
Chapitre 10 Le retour d'expérience des réacteurs de recherche français
}

L'exploitation du retour d'expérience est essentielle pour le maintien et l'amélioration du niveau de sûreté de toutes les installations nucléaires. Pour les réacteurs de recherche, ce retour d'expérience s'appuie sur :

- les enseignements tirés d'événements ${ }^{260}$ survenus dans les installations nucléaires au niveau international, qu'il s'agisse de réacteurs de recherche, de réacteurs électronucléaires ou d'autres types d'installations ;

- l'analyse détaillée (jusqu'à l'identification des causes profondes) des événements survenus sur les réacteurs de recherche français, avec la définition et la mise en œuvre de dispositions permettant d'en éviter le renouvellement ;

- le partage d'enseignements entre les exploitants des réacteurs de recherche et plus largement avec d'autres acteurs, y compris au plan international (notamment sous l'égide de l'AIEA).

260. L'expression adoptée dorénavant en France est « événement », l'expression « incident » faisant plutôt référence aux conditions de fonctionnement de deuxième catégorie de l'analyse de sûreté déterministe. Une guide de l'ASN diffusé le 21 octobre 2005 distingue ainsi les « événements intéressants » et les « événements significatifs ». Les « événements intéressants » (sous-entendu intéressant la sûreté, la radioprotection ou l'environnement) sont ceux qui ont une « importance immédiate qui ne justifie pas une analyse individuelle mais qui peuvent présenter un intérêt dans la mesure où leur caractère répétitif pourrait être le signe d'un problème nécessitant une analyse approfondie « la protection des intérêts » et les « événements significatifs ». 
Concernant les enseignements qui ont été tirés d'événements survenus dans les installations nucléaires au niveau international, trois d'entre eux peuvent être soulignés :

- tout d'abord la nécessité de la prise en compte d'accidents de réactivité - dont l'accident de type BORAX - pour la conception des réacteurs de recherche français, compte tenu des accidents de ce type survenus dans le monde (voir les chapitres 4 et 8 du présent ouvrage) ;

- les interrogations qui ont fait suite à l'accident de Tchernobyl, survenu en 1986, concernant la bonne maîtrise des inhibitions de protections et des raccordements provisoires (« shunts »). Ces interrogations, poussées par l'IPSN, ont notamment conduit à la suppression de certaines possibilités d'inhibition d'actions de sécurité et à une meilleure protection des accès aux dispositifs de réglage des seuils de sécurité de réacteurs de recherche ;

- les interrogations qui ont fait suite à l'accident survenu en 2011 à la centrale nucléaire de Fukushima Daiichi, qui ont conduit aux « évaluations complémentaires de sûreté » (ECS) dont il sera question paragraphe 10.2.

Pour les événements de type anomalie ou écart détectés lors de l'exploitation des réacteurs de recherche français, au-delà de leur identification, déclaration à l'ASN lorsqu'ils relèvent de la catégorie des événements dits significatifs ${ }^{261}$ et traitement qui suit le processus commun aux INB -, un partage d'expérience est effectué dans des instances ad hoc entre les exploitants et leurs chefs d'installation. De son côté, l'IRSN effectue, outre l'analyse de chaque événement et des dispositions proposées par l'exploitant concerné pour en éviter le renouvellement, une analyse dite de deuxième niveau, plus globale et transverse entre les différentes INB (réacteurs de recherche, réacteurs de puissance, installations du cycle du combustible).

Contrairement au cas des réacteurs de puissance, les enseignements tirés d'un événement ayant affecté un réacteur de recherche sont moins immédiatement transposables à d'autres réacteurs de recherche étant donné la diversité des conceptions et des technologies de ces réacteurs. Toutefois, l'analyse de certains événements, concernant par exemple des équipements de levage, des dispositifs de mesure, des " baies » de contrôle-commande, des filtres ou des pièges à iode, ou encore des dispositions organisationnelles peut apporter des enseignements utiles pour plusieurs installations. Ainsi, la découverte en 2012 du mauvais positionnement d'un tube de prélèvement d'air $^{262}$ dans la cheminée du réacteur ORPHEE a conduit l'Autorité de sûreté nucléaire à alerter l'ensemble des exploitants d'INB à ce sujet. Quelques autres préoccupations communes aux réacteurs de recherche ont fait l'objet de règles fondamentales de sûreté spécifiques (dispositifs d'épuration équipant les systèmes de ventilation, protection contre les risques d'incendie - voir le paragraphe 6.2).

Le retour d'expérience de fonctionnement des réacteurs de recherche permet également d'apprécier les évolutions des bilans dosimétriques des travailleurs impliqués

261. Les critères de déclaration des événements significatifs sont été spécifiés dans un guide de l'ASN en date du 21 octobre 2005.

262. Prélèvement pour la mesure du tritium. 
dans les installations sur plusieurs années et de définir si besoin des actions d'amélioration en vue de poursuivre la réduction des doses reçues par le personnel, notamment dans le cadre de l'application des principes de radioprotection.

Il est à rappeler ici (voir le paragraphe 4.1 du présent ouvrage) que, en relation avec les exploitants des réacteurs de recherche français, l'IRSN transmet chaque année à l'AIEA, en tant que coordinateur pour la France, des fiches sur les événements marquants qui ont pu survenir dans ces réacteurs et dont les enseignements méritent d'être partagés au plan international. Ces fiches sont intégrées dans la base de données du système IRSRR qu'exploite l'AIEA qui rassemble l'ensemble des fiches transmises par les différents pays. La base IRSRR constitue une source d'informations et de connaissances accessibles aux exploitants $^{263}$, qui peuvent en tenir compte, notamment dans le cadre des réexamens de sûreté de leurs installations.

Enfin, il convient de rappeler, comme cela a été indiqué au paragraphe 6.1, que les exploitants rendent publics les événements significatifs survenant dans leurs installations, ainsi que l'analyse qu'ils en ont faite et les dispositions prises pour en éviter le renouvellement, cela conformément aux dispositions de la loi TSN.

\subsection{Tendances, quelques événements marquants et leur traitement}

\subsubsection{Tendances}

La base de données des événements, tenue à jour par l'IRSN depuis juin 1972, comportait (fin juin 2018) 1515 événements ayant affecté les réacteurs de recherche français, avec leur classement dans l'échelle INES. Cela correspond à trois événements en moyenne par réacteur et par $a^{264}$. Les événements sont répertoriés dans les rapports annuels (publics) de l'ASN.

Quelques grandes familles d'événements et des tendances peuvent être dégagées :

- des événements non spécifiques des réacteurs de recherche :

- découvertes de corps étrangers dans des éléments combustibles,

- fonctionnements défectueux ou endommagements de composants (éléments combustibles, structures internes, circuits primaires, mécanismes des absorbants, soupapes de sûreté, groupes électrogènes, engins de levage...), pouvant aller jusqu'à la fusion de combustible (SILOE, 1967),

263. En tout état de cause, il appartient aux coordinateurs nationaux de transmettre aux exploitants de leurs pays les fiches d'incidents des bases IRSRR, IRS..

264. La moyenne pour les réacteurs à eau sous pression du parc électronucléaire, installations bien plus complexes que les réacteurs de recherche, est d'environ 13 événements (significatifs) par réacteur et par an (événements touchant la sûreté et la radioprotection). Il peut être utile ici de souligner que le nombre d'événements survenus dans une installation nucléaire ne constitue pas, à lui seul, un indicateur du niveau de sûreté de cette installation. En revanche, l'analyse approfondie de chaque événement est une source fondamentale d'enseignements. 
- fuites de piscines ou de capacités (cela a été tout particulièrement le cas pour le réacteur SILOE, de 1965 à 1986), infiltrations d'eau dans des locaux d'entreposage de matières fissiles,

- défauts d'isolement électrique, pertes de sources électriques externes,

- dysfonctionnements de systèmes de ventilation et pertes de dépression dans des locaux, efficacités insuffisantes de pièges à iode,

- mauvais réglages de seuils de sécurité, non-respects de règles de conduite ou d'exploitation (par exemple des limites fixées pour prévenir les risques de criticité, des conditions d'accès dans les zones contrôlées, des fréquences de contrôles en service),

- des événements en rapport avec des spécificités de conception des réacteurs de recherche : chutes d'objets dans les piscines, fuites de circuits d'eau lourde, rejets de tritium (par les cheminées ou dans les sols) par les installations de détritiation, anomalies concernant des clapets de convection naturelle... ;

- des événements liés à des équipements spécifiques, comme des anomalies relatives aux "doigts de gant » des canaux neutroniques (ORPHEE, RHF) ou aux « sources froides » (ORPHEE, RHF). Ainsi, le RHF a connu une corrosion sous irradiation, décelée à partir de 1972, de la partie interne des doigts de gant en alliage d'aluminium (AG3NET), plus précisément au niveau des « bouchons collimateurs ». Ces derniers se sont grippés par une corrosion due à la formation d'acide nitrique résultant d'un mécanisme de radiolyse de l'air ${ }^{265}$ sous flux neutronique (ce qui a conduit à remplacer l'air par de l'hélium dans les doigts de gant et, lors des remplacements successifs de doigts de gant, par l'installation de nouveaux doigts de gant en Zircaloy). De même, au mois d'août 1988, une erreur a conduit à l'éclatement des disques de rupture protégeant la " source froide » verticale du RHF contre les surpressions et au relâchement, à la cheminée de l'installation (de $45 \mathrm{~m}$ de hauteur), d'environ $9 \mathrm{~m}^{3}$ de deutérium contenant quelques dizaines de $\mathrm{TBq}$ de tritium ;

- des événements liés aux dispositifs expérimentaux et à leur exploitation : déclenchements de sécurités propres à ces dispositifs, réactions chimiques avec le sodium ou l'alliage sodium-potassium, explosions de dispositifs... ;

- des irradiations d'opérateurs, des contaminations de piscines, de bâtiments, de locaux ou de personnes. On peut mentionner à ce sujet le déversement dans la piscine du RHF, en 1974, d'antimoine 124 provenant d'une source neutronique de démarrage. In fine - à la suite de nombreux aléas ${ }^{266}$-, il en est résulté un rejet concerté dans l'Isère (réparti entre 1974 et 1975) de 20 TBq de cet élément radioactif ;

- des découvertes de sources ou d'objets irradiants non répertoriés ;

265. Plus exactement une radiolyse des traces d'eau dans l'air.

266. La presse s'en fit l'écho, comme par exemple Sciences \& Avenir n 360 de février 1977. 
- des départs de feu et des incendies, notamment un incendie dans un local d'électronique d'un dispositif de mesure associé aux expérimentations (CABRI, 1979), une déflagration dans une armoire électrique (RHF, 2000).

Concernant les opérations de manutention et les engins de levage, plusieurs événements se sont produits :

- chute d'un groupe motoréducteur de translation d'un pont (OSIRIS, 1975),

- rupture du ruban d'entraînement d'un treuil (ULYSSE, 1975),

- chute d'un emballage de transport, le crochet du pont roulant s'étant détaché du moufle (ISIS, 1976),

- chute d'assemblage (MASURCA, 1977), de réglettes (même réacteur, 1989),

- chute en piscine d'éléments d'une boucle expérimentale (SILOE, 2004),

- rupture d'un câble de palan et chute d'un crochet sur le «plancher-margelle » (RHF, 2012),

- rupture d'une élingue (RHF, 2013).

Les risques associés aux opérations de manutention nécessitent donc une attention particulière $^{267}$. Il convient d'analyser de manière approfondie les différents aspects de sûreté de ces manutentions : fiabilité des engins de levage, cinématique ${ }^{268}$ des manutentions, facteurs organisationnels et humains, dispositions de limitation des conséquences d'une chute...

Par ailleurs, des dysfonctionnements répétitifs des systèmes de ventilation avec perte de la dépression dans différents locaux de l'installation MASURCA se sont produits de 1991 à 2007. Ils résultaient des défaillances de ventilateurs ou de pertes d'alimentation électrique, attribuables au vieillissement de composants. Il en a été tenu compte dans les projets de rénovation de ces réacteurs.

Si, à partir des années 2000 , la fréquence des événements libellés « fausse manœuvre » ou " erreur de manipulation » dans la base de données tend à se réduire, des non-respects d'exigences règlementaires ou de règles d'exploitation ont émergé, en relation avec le renforcement progressif des « référentiels de sûreté » (notamment d'exploitation) des réacteurs de recherche. La fréquence des événements concernant les expériences et les dispositifs associés diminue au fil des décennies, traduisant une meilleure maîtrise.

Il est intéressant de noter l'apparition, à partir des années 2000, d'événements correspondant à l'identification de lacunes ou d'imperfections de la démonstration de sûreté (anomalie d'étude, erreur de transcription opérationnelle d'exigences...), telles que :

- « défaut d'analyse de sûreté-criticité, dans le rapport de sûreté, relative à un coffre contenant des matières nucléaires sans emploi » (MASURCA, 2003) ;

267. Ce constat est également valable pour les réacteurs du parc électronucléaire.

268. On entend par cinématique de manutention les séquences de mouvement en termes de déplacements horizontaux et verticaux, de vitesse de déplacement, de survols... 
- « utilisation du sas matériel accès galerie couronne non encadrée par le référentiel de sûreté »(OSIRIS, 2005) ;

- « formulation incorrecte de la limite de masse [dans un] « laboratoire chaud » lors de la refonte du rapport de sûreté en 2004 » (MASURCA, 2012).

Dans le paragraphe suivant sont développés quelques-uns des événements les plus marquants au plan de la sûreté ayant affecté des réacteurs de recherche français ainsi que les dispositions correctives qui ont été adoptées à la suite de ces événements. Un certain nombre de ces événements ont fait l'objet d'analyses dans le cadre de réexamens de sûreté. Ces événements, anciens pour la plupart, ont été retenus pour leur intérêt pédagogique, concernant plus particulièrement leur déroulement, les phénomènes physiques mis en jeu, les questions de sûreté soulevées et les réponses qui y sont apportées.

\subsubsection{Quelques événements marquants et leur traitement}

\section{Fuites récurrentes d'eau de la piscine du réacteur SILOE (1965-1986)}

La conception retenue pour assurer l'étanchéité de la piscine du réacteur SILOE a été remise en cause par des fuites d'eau de cette piscine vers la nappe phréatique, qui se sont produites de 1965 à 1972, puis de nouveau en 1986.

Le génie civil de la piscine du réacteur SILOE comportait deux compartiments :

- un compartiment dénommé « piscine principale », d'un volume de $213 \mathrm{~m}^{3}$, contenant le cœur du réacteur ; c'est cette piscine qui a fait l'objet de fuites ;

- un compartiment dénommé « piscine de travail », d'un volume de $322 \mathrm{~m}^{3}$, disposée en fer à cheval autour de la piscine principale et utilisée notamment pour des entreposages de dispositifs expérimentaux et des interventions sur ceux-ci.

La première fuite d'eau de la piscine principale a été décelée en 1965 grâce au bilan des appoints d'eau effectués pour compenser l'évaporation de l'eau de la piscine. Elle disparut en 1968 et réapparut en 1969. Le repérage d'un chapelet de bulles dans l'eau de la piscine, permit, en 1970, de détecter une fissure entre les carreaux de céramique du fond de cette piscine (séparés par des joints en araldite), à proximité d'un pied du « tabouret » supportant le cœur du réacteur. L'étanchéité fut alors rétablie par la pose d'une plaque en acier inoxydable sur le carrelage, avec un joint en mousse synthétique. Mais la fuite d'eau se manifesta de nouveau en 1971, du fait de la dégradation du joint due aux rayonnements. Un colmatage durable de la fuite d'eau n'a été obtenu qu'en 1972 après la mise en place d'un joint plat en caoutchouc naturel appuyé sur le fond de la piscine par du plomb.

Le volume total d'eau contaminée passé dans la nappe phréatique entre 1965 et 1972 a été estimé de l'ordre de $1500 \mathrm{~m}^{3}$. L'activité totale rejetée par cette voie, due principalement au tritium, a été évaluée de l'ordre de $2,7.10^{11} \mathrm{~Bq}$. Lors des travaux de 
remplacement du « tabouret ${ }^{269}$ » de la grille du cœur du réacteur, effectués en 1977, l'étanchéité de la piscine a été refaite en posant sur le carrelage du fond et sur une hauteur d'environ $75 \mathrm{~cm}$ des parois verticales un revêtement d'une épaisseur de $4 \mathrm{~mm}$ de résine époxy associée à un tissu de laine de verre.

La situation durable de non-étanchéité de la piscine amena notamment le Département de sûreté nucléaire ${ }^{270}$ du CEA à évaluer quels seraient les rejets radioactifs dans la nappe phréatique dans le cas hypothétique où se produirait un accident de type BORAX, en tenant compte de la contamination de l'eau de la piscine (du fait de l'accident) et des transferts de radionucléides par la nappe phréatique jusqu'à l'Isère (délai évalué à 55 jours). Il fut ainsi montré que, même sans épuration de l'eau contaminée de la piscine du réacteur, l'activité ${ }^{271}$ volumique ajoutée dans l'Isère serait dans ce cas faible (de l'ordre de $140 \mathrm{~Bq} / \mathrm{l}$ ). L'étude a néanmoins conduit à examiner des dispositions qui permettraient de limiter les conséquences d'un tel accident avec la piscine non-étanche : épuration de l'eau de la piscine (la mise en route des systèmes de « couche chaude » [voir le paragraphe 7.3.3] et de « fond piscine » permettait de ramener l'activité volumique ajoutée dans l'Isère à $0,7 \mathrm{~Bq} / \mathrm{l}$ ), transfert de l'eau de la piscine dans un bassin de l'installation SILOETTE, pompage d'eau sous le radier, dans des puits...

Cependant, au mois de novembre 1986, un accroissement d'environ $1 \mathrm{~m}^{3}$ des appoints d'eau effectués dans la piscine pour compenser l'évaporation fut à nouveau constaté. Après vérification de l'étanchéité de différents circuits du réacteur, celui-ci fut mis à l'arrêt au début du mois de décembre 1986 afin de procéder à une inspection détaillée de la piscine principale, suspectée d'être à l'origine de la fuite d'eau. Cette inspection nécessita de décharger les éléments combustibles, de vidanger la piscine, de démonter les diverses structures du cœur du réacteur et de déposer le carrelage. Le transfert de l'eau de la piscine dans un bassin prévu à cet effet fut réalisé de façon volontairement lente, afin de mettre à profit la baisse progressive de niveau pour effectuer une décontamination des parois de la piscine et d'éviter ainsi la mise en suspension dans l'air de particules radioactives. Une injection de gaz inerte ${ }^{272}$ sous le radier a alors permis de localiser un trou d'environ $5 \mathrm{~mm}$ de diamètre dans un angle au fond de la piscine, ainsi que deux petites fissures. Cet événement s'est traduit par un rejet d'environ $30 \mathrm{~m}^{3}$ d'eau contaminée essentiellement par du tritium dans la nappe phréatique ; l'activité totale a été estimée à 2,3.10 ${ }^{10} \mathrm{~Bq}$.

Cet événement mit en péril l'avenir de $\operatorname{SILOE}^{273}$, compte tenu du coût élevé des travaux nécessaires pour mettre le réacteur en état. Le CEA prit néanmoins la décision d'engager ces travaux en vue de pouvoir poursuivre l'exploitation et les expérimentations dans ce réacteur et programma l'arrêt définitif du réacteur MELUSINE qui avait été mis en service en 1958 et dont les expérimentations pouvaient être transférées dans l'installation SILOE. Les modifications correspondantes, autorisées par le Service central des installations nucléaires (SCSIN) à l'été 1987, furent importantes. Un cuvelage en acier

269. Qui fut alors remplacé par un ensemble grille-tabouret-caisson.

270. Ancêtre de l'IPSN, ce dernier étant créé en 1976.

271. Due à l'ensemble des produits de fission libérés par la fusion du combustible.

272. Le gaz utilisé était de l'hexafluorure de soufre SF6, non toxique, ininflammable, pratiquement insoluble dans l'eau, d'une grande stabilité chimique et thermique.

273. La presse se fit l'écho de l'avancement de la situation : Le Matin du 16 avril 1987, Le Monde du 23 octobre 1987. 
inoxydable fut installé dans la piscine principale, soudé sur des lattes, également en acier inoxydable, fixées sur les murs de cette piscine. Un espace libre fut ménagé sur le pourtour de la piscine et équipé d'un système de détection et de récupération d'une fuite éventuelle du cuvelage. Concernant la résistance de la piscine à un accident de type BORAX, si l'étanchéité des traversées des canaux neutroniques apparaissait acquise, l'instruction technique mit en évidence une faiblesse de résistance de l'une des parois de la piscine. Cela amena l'exploitant à installer une cuve - dite cuve BORAX - capable d'absorber, en se déformant, une partie de l'énergie qui serait dégagée lors d'un tel accident $^{274}$, afin de protéger le cuvelage et les parois de la piscine. Dans le même but, une plaque en acier inoxydable d'une épaisseur de $20 \mathrm{~mm}$, placée sur des absorbeurs de choc, appelés " plots résilients », fut installée sur le radier et sous la cuve BORAX afin d'assurer la tenue mécanique du radier de la piscine à la pression qui résulterait d'un accident de type BORAX. Des cuvelages furent également installés dans les bacs de désactivation, avec des systèmes de détection et de récupération defuite. Ces différents cuvelages et cuves sont représentés sur la figure 10.1.

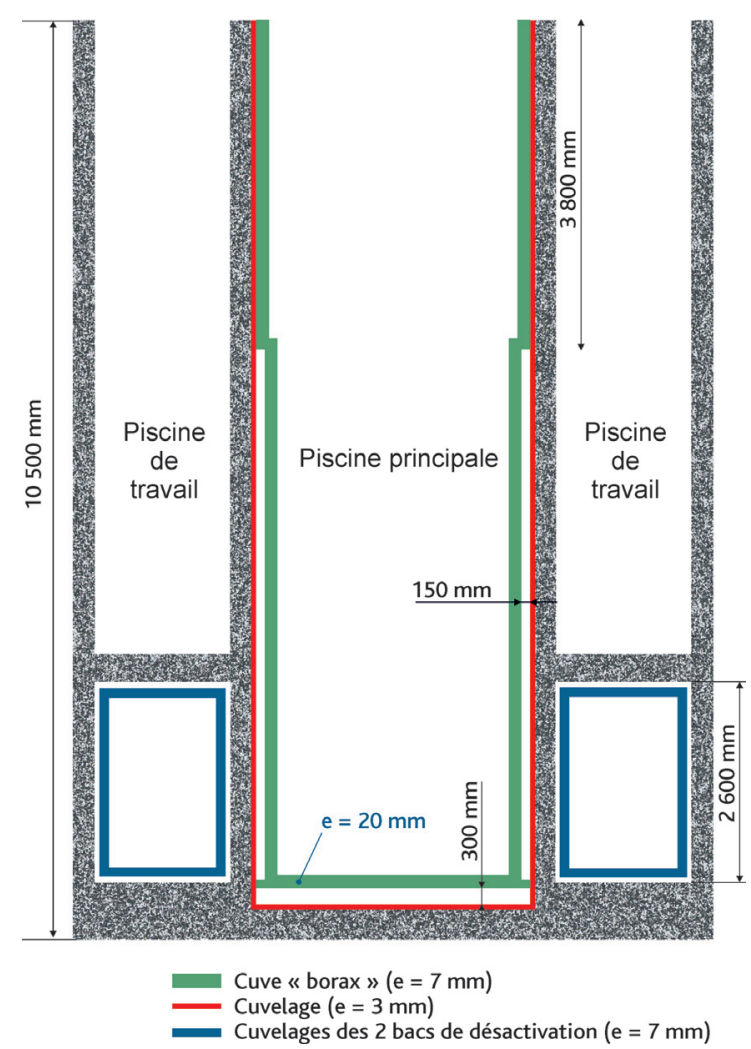

Figure 10.1. Piscines et bacs de désactivation du réacteur SILOE : les cuves et les cuvelages installés en 1987-1988. @ Georges Goué/IRSN.

274. Le reste de cette énergie est dissipé par l'expulsion vers le haut de l'eau. 
De plus, en 1988, deux piézomètres supplémentaires furent installés pour améliorer la surveillance de la nappe phréatique.

Les fuites de la piscine du réacteur SILOE ont eu, ultérieurement, des conséquences pour les opérations de démantèlement de ce réacteur, qui ne sont que succinctement évoquées ci-après.

Les opérations de mise à l'arrêt définitif et de démantèlement du réacteur SILOE ont été soumises à une enquête publique et elles ont fait l'objet d'un décret d'autorisation promulgué au mois de janvier 2005. L'état final, tel qu'envisagé par le CEA, correspondait à un assainissement complet des parois des locaux du réacteur. À cet égard, le CEA s'est fixé en 2008 les objectifs quantitatifs ${ }^{275}$ suivants :

- une « valeur résiduelle modélisée acceptable » (VARMA) théorique d'activité, en deçà de laquelle les structures pourront être considérées comme conventionnelles, de $1 \mathrm{~Bq} / \mathrm{g}$ de béton pour les émetteurs $\beta \gamma$, hors tritium, et de $100 \mathrm{~Bq} / \mathrm{g}$ pour le tritium ;

- pour les émetteurs $\beta \gamma$ hors tritium, un critère de décision de fin de l'assainissement, sur la base des mesures effectuées pour quelques radionucléides, de 0,4 Bq/g, inférieur à la valeur précédente et intégrant une marge supplémentaire pour tenir compte notamment des incertitudes de mesure.

À cette date, la stratégie d'assainissement du radier de la piscine du réacteur et des sols sous-jacents n'avait pas été définie, dans l'attente du retrait de la piscine pour pouvoir déterminer la contamination de ces éléments.

En 2010, le CEA proposa le retrait d'une partie du radier sur toute son épaisseur, et sur une surface couvrant la totalité de la piscine principale. En outre, compte tenu des incertitudes sur le cheminement des fuites dans l'épaisseur du radier, il se proposa de mener des investigations sur les parois verticales mises à nu dans l'épaisseur du radier, dans le but de vérifier l'absence de contamination au niveau des différentes voies possibles de fuite dans le béton, le long des fers à béton ainsi que des gaines de câbles de précontrainte et éléments en feutre bitumineux assurant l'étanchéité.

En 2011, le CEA fit part à l'ASN de la présence d'une contamination dans l'épaisseur du radier au niveau des câbles de précontrainte et dans une partie des terres situées sous le radier (localement, l'activité massique atteignait $25 \mathrm{~Bq} / \mathrm{g}$ ). À ce stade, l'assainissement du radier de SILOE n'avait donc pas permis d'atteindre les objectifs de propreté initialement visés. De son côté, l'ASN était défavorable à la gestion de cette situation par la mise en œuvre d'une "servitude d'utilité publique ${ }^{276}$ ». Par ailleurs, l'assainissement complet nécessitait un traitement en profondeur pouvant affecter la tenue mécanique du bâtiment du réacteur. C'est pourquoi le CEA décida finalement de procéder à la destruction complète du dôme et des murs cylindriques du

275. Dans le « dossier d'information relatif à l'assainissement des structures » (DIRAS) en vue du déclassement.

276. Une servitude d'utilité publique est une servitude administrative qui est annexée au plan local d'urbanisme, conformément à l'article L 126-1 du code de l'urbanisme français. Une étude d'impact y est associée. 
bâtiment du réacteur ainsi que des bâtiments annexes, avant de procéder au retrait complet du radier et des terres contaminées. Les travaux correspondants sont illustrés par la figure 10.2 .
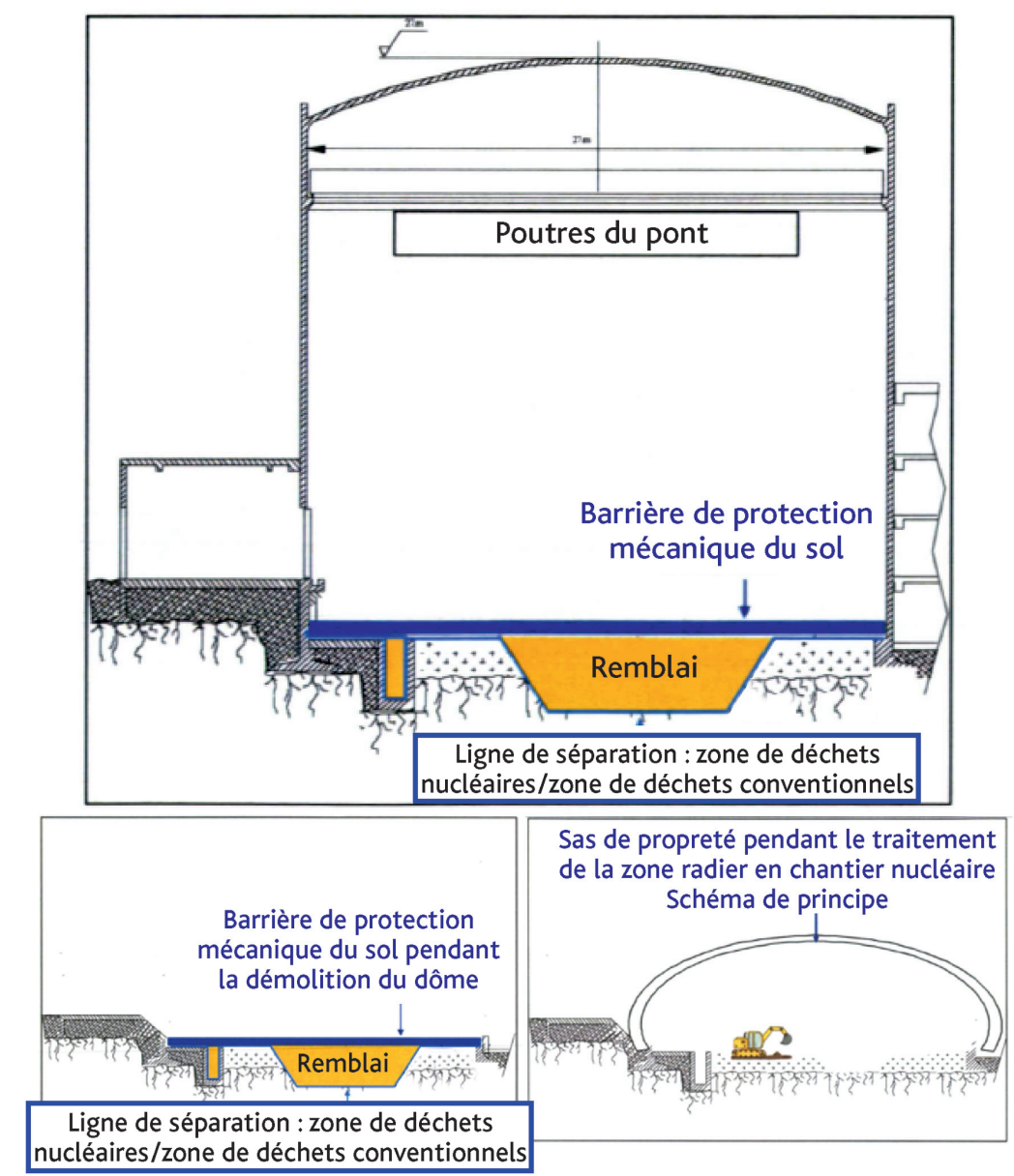

Figure 10.2. Quelques-unes des étapes de la déconstruction du réacteur SILOE (années 2010). @ DR.

Une excavation des terres situées sous le radier du réacteur SILOE a été nécessaire sur une hauteur de $1,8 \mathrm{~m}$.

Le décret de mise à l'arrêt définitif et de démantèlement du réacteur SILOE fut modifié ${ }^{277}$ afin de tenir compte des nouveaux travaux d'assainissement qui entraînèrent un allongement de la durée des opérations. Le réacteur SILOE a été déclassé par une décision de l'ASN en date du 8 janvier 2015, homologuée par un arrêté du ministre en charge de la sûreté nucléaire en date du 12 février $2015^{278}$. 


\section{Fusion de plaques de combustible dans le réacteur SILOE $(1967)^{279}$}

Une fusion partielle de six plaques de combustible appartenant à un élément de contrôle $^{280}$ s'est produite le 7 novembre 1967 dans le réacteur SILOE, au cours d'une montée en puissance à 42,3 MW effectuée dans le cadre d'essais préparatoires à un fonctionnement nominal du réacteur porté à $30 \mathrm{MW}^{281}$. Ces essais, autorisés par la $\mathrm{CSIA}^{282}$, visaient à déterminer notamment les marges disponibles, en termes de puissance, par rapport au phénomène de " redistribution de débit » dans les éléments combustibles (phénomène précisé plus loin). À la puissance de $42,3 \mathrm{MW}$, une baisse brutale de la puissance d'environ $7 \mathrm{MW}$ en une seconde fut observée, suivie d'une baisse plus lente jusqu'à une stabilisation, 20 secondes plus tard, à $20 \mathrm{MW}$. Le réacteur fut arrêté manuellement 26 secondes plus tard, par la chute des deux éléments de sécurité du réacteur. Une augmentation rapide des débits de dose par rayonnement fut ensuite observée (sur une chambre de mesure immergée, jusqu'à $1000 \mathrm{rad} / \mathrm{h}$, et sur une autre chambre de mesure, située au-dessus de l'eau de la piscine, jusqu'à une valeur de $220 \mathrm{rad} / \mathrm{h}$ ), ce qui entraîna l'évacuation du bâtiment du réacteur et des bâtiments annexes, ainsi que l'utilisation des pièges à iode du système de ventilation de secours.

Cet événement s'est traduit par la fusion de $187 \mathrm{~g}$ d'alliage d'uranium et d'aluminium (enrichi à $93 \%$ en uranium 235), correspondant à une masse de 36,8 g d'uranium 235 dont $18 \mathrm{~g}$ ont été relâchés dans le circuit primaire. Le complément a été retrouvé sous la forme d'un magma au pied de l'élément de contrôle (figure 10.3).
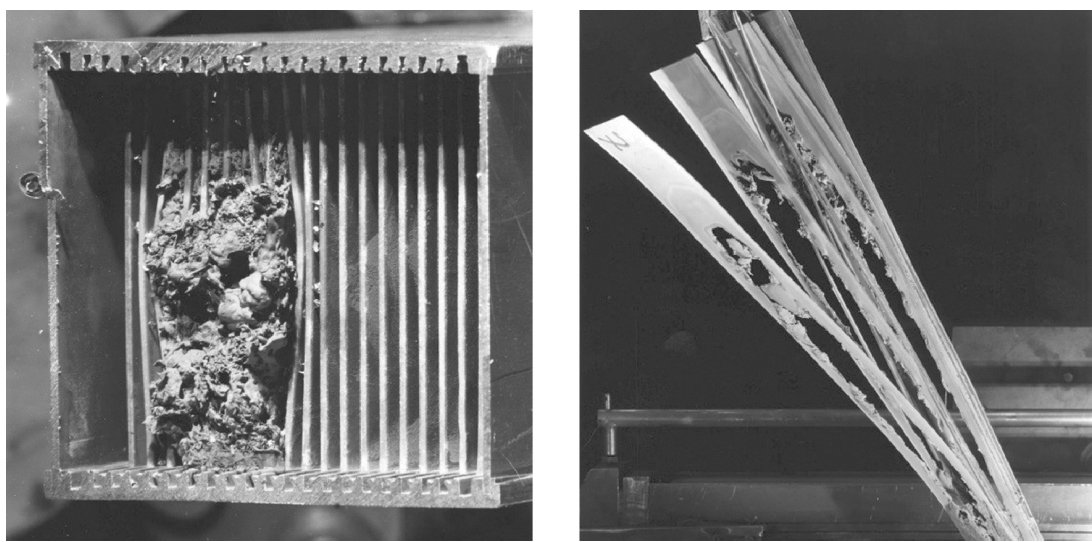

Figure 10.3. Deux vues des plaques fondues de l'élément de contrôle dans le réacteur SILOE. (C) ILL.

Environ $74 \mathrm{TBq}$, principalement de gaz rares, ont été rejetés par la cheminée du réacteur dans les deux journées qui ont suivi l'événement. Aussi bien la contamination du bâtiment du réacteur que celle du site ont été négligeables, du fait du faible taux de

279. Revue Contrôle $\mathrm{n}^{\circ} 128$, avril 1997, ASN.

280. Élément (combustible) contenant des plaques absorbantes de contrôle de la réactivité.

281. Depuis sa mise en service en 1963 , le réacteur fonctionnait à la puissance nominale de 15 MW.

282. Commission de sûreté des installations atomiques. 
combustion (4\%) de l'élément concerné. Le combustible dispersé dans le circuit primaire a été récupéré dans les bacs de désactivation à l'occasion des visites de ces bacs effectuées en 1970 et 1971.

La cause de la fusion n'a pas été clairement établie. À cet égard, les résultats des investigations menées par l'exploitant à la suite de cet événement, qui ont notamment consisté à mesurer les températures des gaines d'éléments de contrôle du même type, placés à l'endroit où se trouvaient les plaques combustibles fondues, pour des puissances allant de 0 à $39 \mathrm{MW}$, semblaient permettre d'écarter l'hypothèse d'une redistribution de débit $^{283}$; en effet, l'extrapolation de ces mesures à une puissance de 42,3 MW conduisait, pour les plaques fondues, à une température au point chaud des gaines inférieure à celle correspondant à l'ébullition locale $\left(116^{\circ} \mathrm{C}\right.$ à comparer à $\left.128^{\circ} \mathrm{C}\right)$. En outre, la plaque la plus chaude n'a pas fondu.

Des écailles de peinture sèche, en provenance des structures situées au-dessus de la piscine, ayant été trouvées à plusieurs reprises sur des éléments du cœur, l'exploitant a finalement attribué la fusion des plaques à un bouchage partiel de plusieurs canaux de refroidissement de l'élément de contrôle concerné.

Les améliorations réalisées à la suite de cet événement ont consisté à :

- remplacer les structures en tôle peinte surplombant le cœur, utilisées pour le cheminement de câbles et de flexibles des dispositifs expérimentaux, par des structures en acier inoxydable ;

- doubler le circuit d'extraction de secours de l'installation, afin d'accroître la fiabilité d'un tel système important pour la sûreté ;

- installer des circuits permettant des prélèvements d'air au-dessus de la piscine et des prélèvements d'eau dans cette piscine, depuis l'extérieur du bâtiment du réacteur ;

- installer dans la salle de contrôle une commande du circuit d'épuration de l'eau de la piscine.

L'événement ne nécessita pas de décontamination. Le réacteur fut autorisé à redémarrer quelques jours après.

\section{- Éclatement du dispositif expérimental d'irradiation AQUILON dans SILOE (1979)}

L'éclatement du dispositif expérimental d'irradiation AQUILON s'est produit dans le réacteur SILOE le 10 avril 1979. Ce dispositif - apparenté aux « bouilleurs ${ }^{284}$ " utilisés pour des essais relatifs aux combustibles des réacteurs à eau légère - était placé à la

283. Phénomène qui peut résulter d'une surchauffe et d'une ébullition locales, se traduisant par une modification des débits d'eau entre les plaques. Différents essais, réalisés dans le réacteur CABRI, avaient déjà montré que, en général, une redistribution de débit est accompagnée d'oscillations de puissance dues à l'ébullition locale et à l'effet de vide correspondant; or de telles oscillations n'ont pas été observées lors de l'événement ici rapporté.

284. La pression avait été portée à 130 bars dans le dispositif AQUILON au lieu de 78 bars dans les «bouilleurs ». 
périphérie du cœur et contenait un crayon de combustible d'oxyde d'uranium $\left(\mathrm{UO}_{2}\right)$ irradié (uranium enrichi à $4,7 \%$ en isotope 235) (figure 10.4). L'éclatement du dispositif a conduit au relâchement d'oxyde d'uranium et de produits de fission dans la piscine du réacteur et au relâchement de gaz rares et d'iodes dans le bâtiment du réacteur et dans l'environnement.

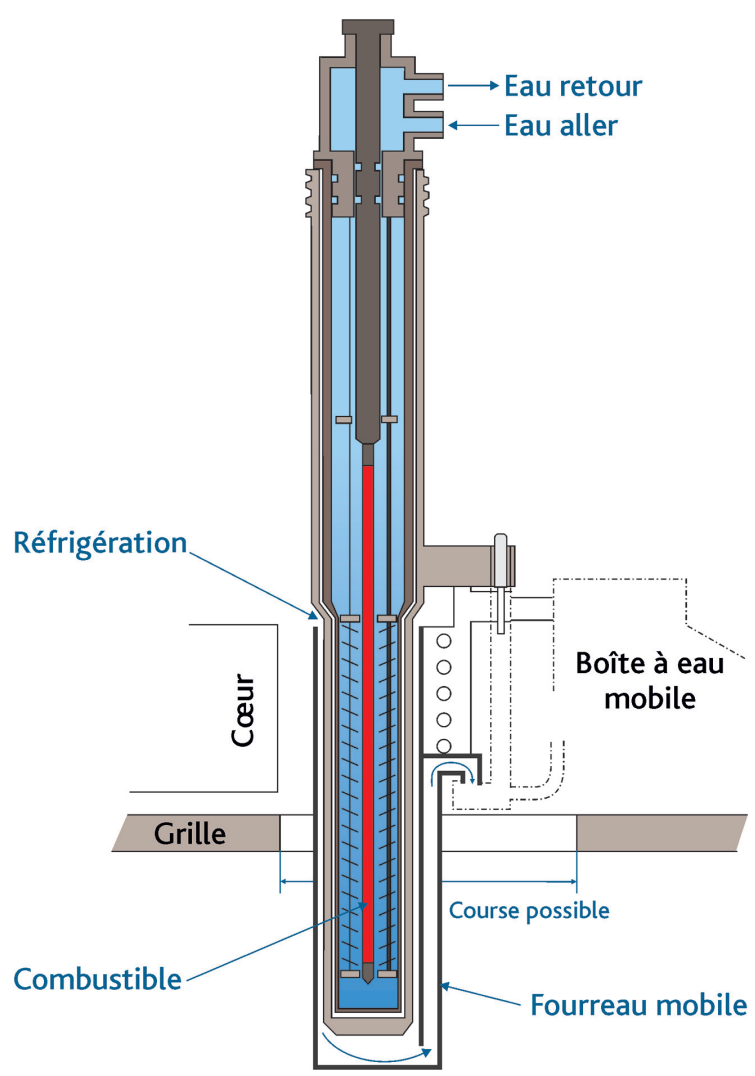

Figure 10.4. Vue en coupe du dispositif AQUILON. C Georges Goué/IRSN.

Dans le dispositif, l'eau était pratiquement statique ${ }^{285}$, sa pression était ajustée en fonction de la chaleur dégagée par le crayon, de façon à obtenir une ébullition nucléée. La vapeur surchauffée produite était dirigée par des couronnes de guidage du crayon à la paroi froide du dispositif, où elle se condensait. La paroi externe du dispositif était refroidie par une circulation d'eau de la piscine. À cette fin, un fourreau cylindrique, disposé autour de la partie basse du dispositif située au droit du cœur du réacteur SILOE, délimitait un canal de refroidissement (d'une " épaisseur » de $2 \mathrm{~mm}$ ). Ce dernier était raccordé au circuit de refroidissement du réacteur par l'intermédiaire d'une « boîte à eau » mobile (permettant le rapprochement ou l'éloignement du dispositif par rapport au cœur).

285. Le dispositif n'était pas équipé d'une pompe de circulation d'eau, de sorte que seule une convection naturelle pouvait s'y établir. 
Le fourreau, d'une masse d'environ $10 \mathrm{~kg}$, prenait appui sur la « boîte à eau », mais n'était pas verrouillé mécaniquement à cette dernière.

L'arrêt du réacteur pouvait être déclenché automatiquement par un certain nombre de sécurités associées à la boucle (en cas de rupture du tube sous haute pression ou en cas de niveau bas de l'eau dans le pressuriseur, ou encore de température élevée dans la partie basse du dispositif).

Le 10 avril 1979, à 9 h 17, le dispositif était en position reculée par rapport au cœur depuis environ 17 heures (les sécurités du dispositif étaient inhibées par des « shunts ») et le réacteur était en fonctionnement. Trois agents de manutention procédaient au retrait du dispositif à l'aide d'un pont roulant.

À $9 \mathrm{~h} 20$, l'ordre d'évacuation du hall du réacteur a été donné depuis la salle de contrôle, à la suite du dépassement du deuxième seuil de débit de dose, réglé à $0,25 \mathrm{mGy} / \mathrm{h}$, sur une chambre de mesure de rayonnement $\gamma$ située au-dessus de la piscine. Cinq minutes plus tard, une explosion a été perçue en salle de contrôle et les deux absorbants de sécurité ont chuté. Pratiquement au même moment, les seuils d'alarme et de sécurité de nombreuses voies de mesure des rayonnements ont été franchis. L'évacuation du bâtiment a été ordonnée (le débit de dose atteignant $0,4 \mathrm{mGy} / \mathrm{h}$ dans la salle de contrôle) et réalisée après que les opérateurs eurent commandé la chute des trois absorbants de contrôle, l'arrêt des pompes primaires et la mise en service de l'extraction d'air de secours (ventilation du hall du réacteur avec pièges à iode).

Les constatations suivantes ont été faites lors des investigations qui ont suivi :

- le fourreau du dispositif portait des traces d'échauffement et était percé de deux trous ;

- le crayon de combustible d'essai était partiellement fondu ;

- la partie supérieure de la plaque externe d'un élément combustible situé près de la « boîte à eau » du dispositif était déformée, sans rupture de gaines.

Environ $190 \mathrm{~g} \mathrm{~d}^{\prime} \mathrm{UO}_{2}$ (7,5 g d'uranium 235) ont été expulsés du dispositif dans la piscine.

L'explosion du dispositif a conduit notamment à une libération de 18,5 TBq de produits de fission dans la piscine. Les rejets dans l'environnement ont été estimés à

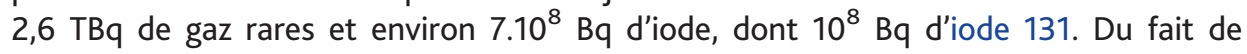
l'évacuation rapide du hall du réacteur par les trois agents de manutention avant l'explosion, la dose reçue par l'agent le plus exposé a été limitée à 1,8 mGy. L'activité volumique maximale des rejets gazeux par la cheminée du réacteur a été de $1,48.10^{5} \mathrm{~Bq} / \mathrm{l}$ (xénon). Les mesures effectuées dans l'environnement, sous le vent, n'ont pas mis en évidence d'activité due aux aérosols ou aux iodes supérieure au bruit de fond naturel.

La cause de l'événement a été déterminée : un entraînement du fourreau s'est produit lors du retrait du dispositif AQUILON, qui a eu pour conséquences :

- d'interrompre le refroidissement du dispositif (désaccouplement de la liaison entre le fourreau et la « boîte à eau ») ; 
- de supprimer le guidage d'eau de refroidissement normalement assuré par le fourreau, jusqu'à ce que la partie inférieure du dispositif soit complètement sortie du cœur du réacteur.

Par ailleurs, il n'a pas été possible d'affirmer que la soupape du pressuriseur du dispositif avait bien fonctionné.

Tous les éléments combustibles du cœur du réacteur SILOE ont été contrôlés à l'aide d'un dispositif mobile de détection de rupture de gaine. Les six éléments qui avaient été les plus proches du dispositif AQUILON ont été remplacés. Le réacteur a été autorisé à redémarrer par le Service central de sûreté des installations nucléaires, après un arrêt de dix jours consacrés pour l'essentiel à l'épuration de l'eau de la piscine. Toutefois, l'utilisation des dispositifs de type «bouilleur » est restée suspendue pour notamment faire le point sur la situation de ces dispositifs par rapport à la règlementation des appareils à pression.

Les dispositions générales prises par le CEA après l'événement - à la suite de la réunion 11 juillet 1979 de la Commission de sûreté des piles - ont porté notamment sur :

- l'interdiction du « shunt » des sécurités associées aux dispositifs expérimentaux durant leur retrait hors d'un cœur ou leur introduction dans celui-ci ;

- le verrouillage des fourreaux sur les «boîtes à eau » ;

- une rédaction plus complète et plus précise des procédures pour l'introduction en cœur, ou le retrait, de divers dispositifs, réacteur en fonctionnement ou à l'arrêt.

\section{Sous-estimation de la puissance de fonctionnement du RHF (1971-1990)}

Une anomalie de sous-estimation, permanente, de la puissance réelle de fonctionnement du RHF, de l'ordre de $10 \%$, a été découverte au mois de janvier 1990 à la suite de la vérification de certains paramètres de fonctionnement du réacteur. Dès ce constat, l'exploitant a réduit cette puissance de fonctionnement, afin de respecter la valeur maximale de 58,3 MWth définie dans les prescriptions techniques qui lui étaient applicables.

Cette anomalie a été déclarée au Service central de sûreté des installations nucléaire le 23 janvier 1990. Elle résultait d'une erreur de calcul du débit d'eau lourde dans le circuit principal du réacteur. La formule utilisée, définie sur la base de mesures effectuées avec de l'eau légère, n'avait pas été corrigée pour tenir compte de la différence de masse volumique entre l'eau légère et l'eau lourde. Le débit réel était en fait supérieur de $10 \%$ au débit calculé. L'erreur avait été également faite lors de la détermination des seuils du système de protection du réacteur, de sorte qu'il n'y eut pas d'alarme ou d'arrêt d'urgence automatique du réacteur.

Le 24 janvier 1990, le Service central de sûreté des installations nucléaires demanda à l'exploitant de maintenir le réacteur à l'arrêt ${ }^{286}$, en soumettant le redémarrage à son autorisation.

286. L'exploitant, qui avait abaissé dans un premier temps la puissance du réacteur, avait finalement pris la décision d'arrêter le réacteur. 
Il apparut que des observations faites précédemment par l'exploitant trouvaient leur explication dans cette sous-estimation de la puissance de fonctionnement. L'exploitant avait en effet observé une consommation de combustible plus rapide que prévue, qui se traduisait par un raccourcissement des cycles de fonctionnement (la position de fin d'irradiation des barres absorbantes était obtenue au bout de 42 jours au lieu des 46 prévus).

Cette anomalie a conduit l'exploitant à revoir totalement les études de physique du cœur du RHF (neutronique et thermohydraulique).

\section{Fusion de combustible dans des crayons du cœur nourricier du réacteur CABRI (2004) 287288}

Dans le cadre des actions menées au titre du réexamen de sûreté associé au projet de modification de l'installation CABRI pour y permettre des essais dans une boucle à eau sous pression, des examens portant sur les crayons les plus chauds du cœur du réacteur ont été réalisés. Les premiers examens non destructifs réalisés sur trois d'entre eux ont alors mis en évidence l'existence de plis sur leurs gaines. Ces plis avaient une amplitude de plusieurs dizaines de micromètres et des pas (distances entre plis) voisins de $10 \mathrm{~mm}-$ correspondant approximativement à la hauteur d'une pastille de combustible - à la partie inférieure des trois crayons. Pour deux des crayons, il a été observé une zone de déformation importante de quelques pour cent au-dessus de la zone des plis.

À la suite de ces observations, une coupe radiale a été réalisée dans la région du maximum de déformation de l'un des crayons. Une macrographie de cette coupe a montré la présence d'une fusion locale au centre du combustible et des traces d'une interaction pastille-gaine. Ces observations constituaient des écarts aux prescriptions techniques du réacteur CABRI et ont fait l'objet d'une déclaration à la Direction de la sûreté des installations nucléaires le 12 janvier 2004.

Comme cela a été indiqué dans le chapitre 5 , le réacteur CABRI a été conçu pour réaliser des excursions de puissance (« pulses » - voir la figure 10.5) à partir d'un fonctionnement en régime permanent de $25 \mathrm{MW}$ maximum. Dans l'objectif d'éviter la fusion du combustible et d'assurer l'étanchéité des gaines des crayons du cœur nourricier, les prescriptions techniques de l'installation imposaient que, pendant ces excursions de puissance, la température maximale du combustible n'excède pas $2400{ }^{\circ} \mathrm{C}^{289}$, toutes incertitudes cumulées, et que la puissance thermique surfacique des crayons ${ }^{290}$ du cœur reste inférieure à $750 \mathrm{~W} / \mathrm{cm}^{2}$ (critères à respecter pour les estimations, par le calcul, préalables aux expériences). Par ailleurs, ces prescriptions techniques autorisaient un fonctionnement du réacteur au-delà de $25 \mathrm{MW}$ jusqu'à 42,5 MW pour une durée cumulée maximale de 23 minutes afin de réaliser des rampes lentes de puissance; dans ces conditions, la température maximale du combustible ne devait pas dépasser $2768^{\circ} \mathrm{C}$,

287. Site ASN, déclarations d'incidents.

288. Synthèse du rapport de l'IRSN sur le redémarrage du réacteur CABRI, en date du 22 janvier 2009, rendue publique.

289. La température de fusion de l'UO $\mathrm{O}_{2}$ est d'environ $2840{ }^{\circ} \mathrm{C}$.

290. Valeur issue d'essais (CAPRI) qui avaient été réalisés dans une boucle au CEA de Grenoble. 


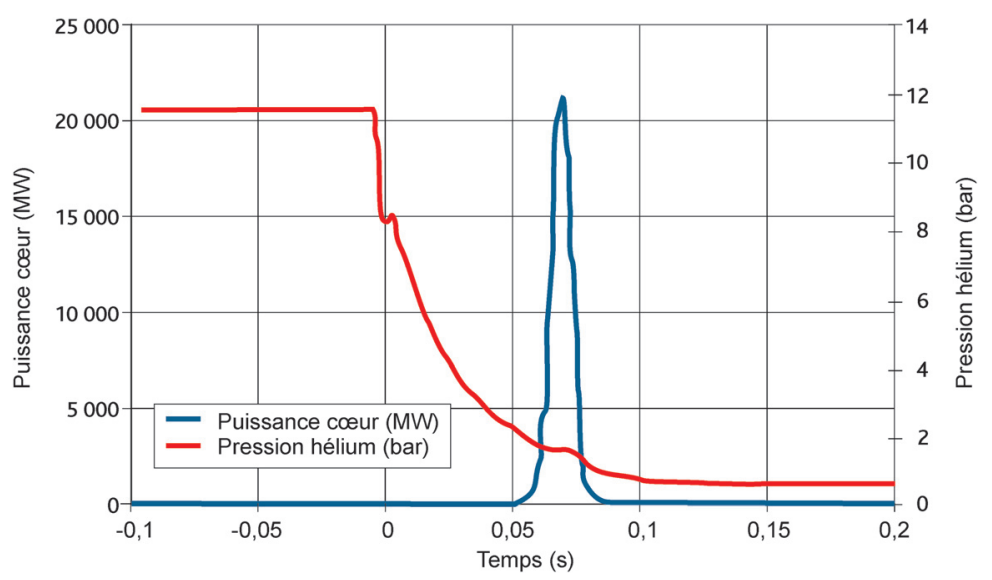

Figure 10.5. Exemple de profil temporel de puissance dans le cas d'un «pulse » de puissance dans le réacteur CABRI. @ Georges Goué/IRSN.

toutes incertitudes cumulées (exigences indiquées dans le rapport de sûreté, justifiées par le calcul).

Depuis la divergence du réacteur CABRI en 1977, environ 500 transitoires rapides de puissance (« pulses ») et 16 rampes lentes de puissance au-delà de $25 \mathrm{MW}$ pendant une durée cumulée de 19,8 minutes avaient été réalisées avec le cœur nourricier. Le taux de combustion moyen du cœur du réacteur était de $88 \mathrm{MWj} / \mathrm{tU}$ (environ 2,3 jours équivalents à pleine puissance [JEPP]).

Le combustible du cœur du réacteur est constitué d' $\mathrm{UO}_{2}$ à faible enrichissement en uranium 235 , sous forme de pastilles empilées dans des gaines en acier inoxydable (de nuance $304 \mathrm{~L}$ selon la norme américaine AISI).

Les dispositions retenues à la conception pour limiter les risques d'assèchement des crayons de combustible lors des transitoires rapides de puissance étaient les suivantes:

- l'adoption d'un jeu radial initial combustible-gaine élevé pour limiter les interactions pastille-gaine ;

- l'adoption d'une pression interne d'hélium à froid suffisante pour écarter, à chaud, le risque de fluage de la gaine sous l'effet de la pression externe ;

- le choix de pastilles d'un type particulier ${ }^{291}$, peu propice à la fracturation sous l'effet d'un gradient thermique radial (conservation de la géométrie initiale des pastilles) ;

- le choix d'un gainage en acier ayant un fort coefficient de dilatation, contribuant à limiter la fermeture du jeu pastille-gaine lors des transitoires de puissance.

291. Par un procédé de frittage différent de celui utilisé pour les pastilles de combustible destinées aux crayons des réacteurs du parc électronucléaire. 
Le bon état du combustible avait été précédemment vérifié par l'examen de crayons parmi les plus chauds du cœur du réacteur, d'abord en 1988 après la réalisation d'environ 200 transitoires rapides, puis en 1991 après un fonctionnement cumulé de près de 10 minutes au-delà de $25 \mathrm{MW}$ en rampes lentes (sur les $23 \mathrm{mn}$ autorisées).

Les investigations ont conduit à attribuer les déformations des gaines et la fusion de combustible à cœur à des transitoires de type rampe lente qui entraînent une déformation en forme de diabolo des pastilles de combustible ; ainsi, en cas d'interaction pastillegaine pendant de tels transitoires, des plis sur la gaine peuvent apparaître (les transitoires rapides de puissance conduisent à une déformation des pastilles en forme de tonneau). Par ailleurs, seules les rampes lentes conduisent à des températures du combustible proches de la température de fusion de celui-ci. Il en a été déduit que les déformations constatées étaient probablement apparues après la dixième minute de fonctionnement au-delà de $25 \mathrm{MW}$.

Les observations faites soulevèrent un certain nombre de questions de sûreté, concernant in fine l'aptitude du cœur nourricier du réacteur CABRI à pouvoir subir les essais futurs prévus (10 transitoires rapides du programme $\mathrm{CIP}^{292}$ ) :

- Quel était l'état du cœur du réacteur dans son ensemble ?

- Quelles dispositions de remise en conformité du cœur devaient être prises ?

- Quelles dispositions renforcées de surveillance en service des crayons de combustible du cœur devaient être prises ?

- La distribution de la puissance dégagée dans le cœur du réacteur était-elle bien connue ?

- Les outils de simulation, utilisés pour vérifier avant chaque nouvel essai que les critères relatifs au combustible et à son gainage seraient respectés, donnaient-ils des résultats fiables?

- Fallait-il envisager des restrictions d'utilisation du réacteur pour les programmes futurs?

Compte tenu de l'implication de l'IRSN dans les recherches menées à l'aide du réacteur CABRI, les dossiers établis par l'exploitant CEA ont fait l'objet, à la demande de l'IRSN, d'un examen par l'organisme belge de sûreté nucléaire AVN (Association Vinçotte-Nucléaire).

$\mathrm{Au}$ vu des observations faites sur les trois crayons mentionnés précédemment, une démarche « d'extension de contrôle 293 » a été appliquée. Des examens non destructifs ont ainsi été également réalisés sur six crayons supplémentaires parmi les 1488 crayons du cœur du réacteur CABRI ; seules de faibles déformations de leurs gaines ont été observées.

292. Cabri International Programme. Voir l'ouvrage « État des recherches dans le domaine de la sûreté des réacteurs à eau sous pression ", Collection sciences et techniques, IRSN/EDP Sciences, 2017.

293. Démarche usuelle qui n'est pas propre aux réacteurs de recherche. 
La remise en conformité du cœur du réacteur a consisté à remplacer quatre crayons (celui qui avait fait l'objet d'une expertise destructive et les trois autres crayons du cœur qui produisaient une puissance analogue à celle produite par le crayon expertisé) par des crayons neufs. Il a été estimé qu'un endommagement des autres crayons du cœur du réacteur n'était pas à craindre du fait qu'ils ne produisaient pas une puissance suffisante.

Par ailleurs, une analyse détaillée des transitoires subis par le cœur du réacteur a permis d'attribuer la fusion de combustible et les déformations associées à un transitoire de type rampe lente réalisé en 1995. Le CEA a alors pris la décision de ne plus faire d'essais de type rampe lente dans le réacteur CABRI.

Les analyses réalisées ont montré que des paramètres importants étaient mal estimés par les différents logiciels de simulation du comportement des crayons de combustible mis en œuvre pour les études de sûreté conduites préalablement à la réalisation des transitoires programmés. Cela explique que la fusion de combustible n'avait pas été anticipée. Un nouvel outil de simulation a alors été mis au point par le CEA (ce sujet est développé au chapitre 11). En outre, s'appuyant sur le fait que nombre de transitoires subis par le cœur du réacteur avaient sollicité les crayons de combustible davantage qu'ils ne pourraient l'être à l'avenir, et ce sans qu'aucune rupture de gaine n'ait été détectée, le CEA a proposé de fonder dorénavant la démonstration de l'absence de rupture de gaine lors d'un transitoire sur la vérification du respect de deux critères associés à la déformation circonférentielle et à la température des gaines. Cela a été accepté compte tenu notamment de la bonne ductilité de l'acier austénitique utilisé pour le gainage des crayons de combustible du réacteur CABRI, peu irradié, ainsi que de la faible quantité de produits de fission accumulés dans ce combustible. À cet égard, l'IRSN a réalisé une étude, lors d'une expertise des dossiers du CEA, pour comparer l'énergie que devrait produire un crayon du cœur du réacteur au cours d'un transitoire rapide pour provoquer l'atteinte des nouveaux critères à l'énergie représentative d'une limite de rupture de gaine déterminée sur la base d'essais effectués aux États-Unis et au Japon ${ }^{294}$; cette étude a montré la cohérence de ces critères pour les transitoires prévus dans le réacteur CABRI (programme CIP).

Finalement, les résultats des calculs réalisés avec le nouvel outil de simulation ont permis d'estimer que les déformations des gaines et les températures des gaines et du combustible ne devraient pas conduire à des ruptures de gaines lors des essais du programme CIP.

En termes de surveillance en service, le programme retenu par le CEA, pour les crayons du cœur du réacteur CABRI, comporte :

- la réalisation d'un « point zéro ». Cette première étape a été réalisée et a consisté à effectuer une profilométrie ${ }^{295}$ de référence des quatre crayons se substituant à

294. Il s'agit des essais SPERT-CDC aux États-Unis et des essais NSRR au Japon, réalisés en 1969 et dans les années 1970 avec différents matériaux de gainage, dont de l'acier inoxydable comme cela est le cas des crayons du cœur nourricier du réacteur CABRI. Ces essais avaient alors conduit à retenir une valeur limite, en transitoires rapides, de $240 \mathrm{cal} / \mathrm{g}$ pour les crayons de réacteurs à eau sous pression.

295. Mesures diamétrales effectuées pour deux azimuts distants de $90^{\circ}$, selon un pas axial serré. 
ceux qui ont subi une fusion locale (situés dans deux assemblages de barres de commande (contrôle) et de sécurité $\left.{ }^{296}[B C S]\right)$;

- la réalisation d'une profilométrie à la fin du programme CIP. Cette seconde étape consistera au déchargement de l'un des deux assemblages BCS contenant les crayons chauds et à l'extraction de l'un d'eux pour la réalisation d'une profilométrie. En cas d'évolution notable (hauteur de pli supérieure à $60 \mu \mathrm{m}$ ), une extension de l'inspection à deux autres crayons chauds de l'assemblage BCS sera engagée. Une évolution mineure du plissement est naturellement attendue car les crayons de substitution (déjà irradiés) n'étaient pas, par définition, à l'emplacement du point chaud. Le « pulse » enveloppe des futurs essais devrait donc engendrer un niveau de plissement de la gaine des nouveaux crayons chauds de l'ordre de 60 à $100 \mu \mathrm{m}$ d'après les calculs prédictifs de thermomécanique.

\section{Irradiation de travailleurs}

Dix-huit événements d'irradiation ${ }^{297}$ de travailleurs se sont produits dans les réacteurs de recherche français depuis 1973 (moins d'un événement de ce type par réacteur et par décennie). Six réacteurs sont concernés dont principalement les réacteurs SILOE, ISIS et OSIRIS ainsi que le RHF. L'évolution du nombre d'événements au cours du temps est présentée sur la figure 10.6 ci-après.

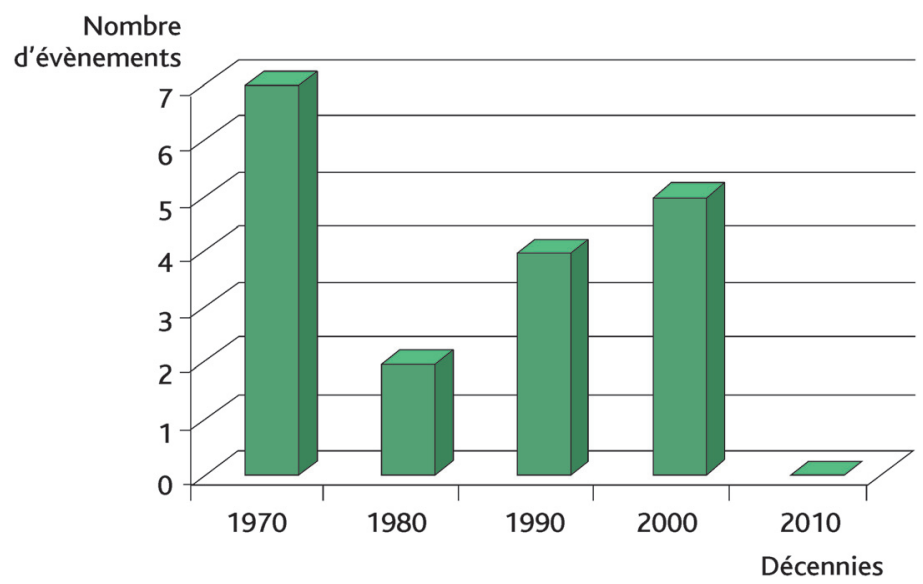

Figure 10.6. Évolution du nombre d'événements d'irradiation de travailleurs au cours des décennies 1970, 80, 90, 2000 et 2010 (2010-2014) dans les réacteurs de recherche français. @ Georges Goué/IRSN.

Sur une durée de 41 années (1973-2014), environ une trentaine de personnes ont subi une irradiation, avec des doses maximales de l'ordre de $40 \mathrm{mSv}$, hormis dans le cas de l'événement survenu le 15 novembre 1979 dans le réacteur SILOE : lors de la dépose d'un

296. Le cœur nourricier du réacteur CABRI dispose de six assemblages, dits assemblages BCS ou simplement BCS, comportant 21 crayons de combustible et 25 tubes recevant les crayons absorbants.

297. Incluant quelques (rares) événements de contamination. 
film photographique sur une plaque activée de dysprosium préalablement irradiée dans l'axe d'un faisceau, un opérateur a reçu une dose évaluée à 140 mSv (rayonnements X, $\beta$ et $\gamma$ ). Depuis 1990, il n'y a plus eu d'irradiation importante de travailleurs (figure 10.7).

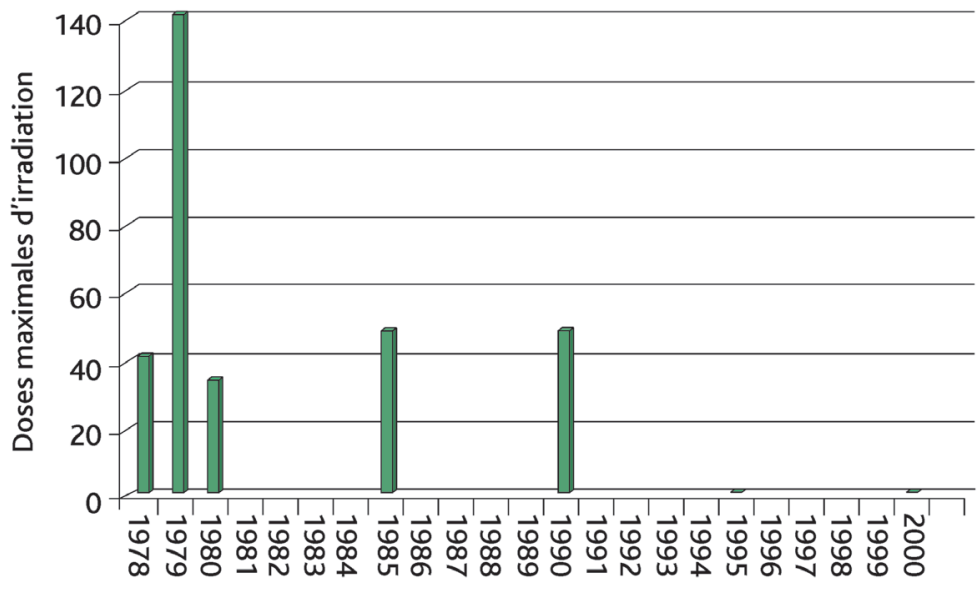

Années

Figure 10.7. Évolution des doses maximales d'irradiation reçues par des opérateurs, en mSv $(1978,1979$, 1980, 1985, 1990) dans les réacteurs de recherche français. ( $)$ Georges Goué/IRSN.

Un événement survenu au mois d'octobre 1985 dans le réacteur SILOE mérite d'être décrit ici, du fait qu'il illustre les risques que peuvent générer des activités multiples menées de façon simultanée dans une installation en général. Le développement du film dosimètre d'un intervenant extérieur, qui était occupé à des travaux de réfection de l'étanchéité de l'enceinte du réacteur (structure métallique), a indiqué une dose reçue de $47 \mathrm{mSv}$. Les investigations menées ont montré que cet intervenant avait travaillé sur le dôme du réacteur (enceinte métallique), au droit d'une " cellule chaude » située dans le hall du réacteur, en même temps qu'une source de cobalt 60 de près de 100000 Curies (destinée à la médecine) était manutentionnée dans cette cellule par un moyen de levage situé sur le toit de la cellule et dont le câble traversait l'épaisseur de béton par un trou de faible diamètre. Lors de cette opération, un faisceau très étroit de rayonnement ionisant était dirigé vers le dôme, affectant une faible surface d'environ $1 \mathrm{~m}^{2}$. Les investigations permirent également d'attribuer cette dose de $47 \mathrm{mSv}$ uniquement au temps $(20 \mathrm{mn})$ pendant lequel la source avait été extraite de la cellule. La procédure de retrait d'une source de la " cellule chaude » fut ensuite complétée afin de requérir, préalablement à un tel retrait, la vérification de l'absence de travaux sur le dôme du réacteur SILOE.

Plusieurs événements liés à l'utilisation de canaux neutroniques ont concerné le réacteur ORPHEE et le RHF, avec une plus forte récurrence pour le RHF, s'expliquant par le plus grand nombre d'expériences de physique qui sont menées simultanément dans ce réacteur (40 dans le RHF contre 25 dans ORPHEE), et de leurs durées, en moyenne plus courtes pour le RHF. 
Deux événements de ce type, l'un survenu en juin 1989 dans le réacteur ORPHEE - qui aurait pu conduire à une irradiation accidentelle de personnes -, l'autre au mois d'août 1990 dans le RHF - qui a conduit à une (faible) irradiation de deux physiciens et de deux agents d'exploitation (dose maximale de l'ordre de $20 \mathrm{mSv}$ ) - méritent d'être décrits ici car ils illustrent les difficultés, évoquées au paragraphe 7.2.3, résultant de la présence de plusieurs populations opérant au sein de réacteurs de recherche, à savoir d'une part le personnel d'exploitation, d'autre part les expérimentateurs. Ces deux événements ont conduit les exploitants concernés et l'IPSN à mener une analyse approfondie concernant :

- les dispositions techniques d'obturation des "faisceaux sortis de neutrons »,

- les facteurs organisationnels et humains.

Les dispositions techniques d'obturation reposent notamment sur la présence d'obturateurs physiques (un obturateur dit " de tête ", placé en amont du faisceau de neutrons, et un obturateur secondaire ${ }^{298}$, qui peuvent être tous les deux de technologies ${ }^{299}$ diverses) permettant d'interrompre le faisceau de neutrons et de rétablir la continuité de la protection biologique du réacteur. Ces obturateurs qui constituent des éléments importants pour la sûreté font l'objet de vérifications périodiques. Les deux événements de 1989 et 1990 ont mis en évidence des défauts dans la conception des obturateurs (obturateur déséquilibré ${ }^{300}$ par une petite fuite d'air comprimé dans le cas du réacteur ORPHEE, entraînant une ouverture inopinée du faisceau ; obturateur isolé [pour des raisons d'interventions] de son alimentation en air comprimé dans le cas du RHF d'où l'impossibilité d'interrompre le faisceau). Diverses améliorations ont été mises en œuvre, notamment sur les circuits d'air comprimé de commande des obturateurs.

En matière de facteurs organisationnels et humains, l'événement survenu dans le réacteur RHF a conduit l'IPSN à mener, en accord avec l'exploitant, sa propre analyse. Celle-ci a conduit aux constats suivants :

- il existait un contexte particulier de situation contrainte pour les physicienschercheurs à l'Institut Laue-Langevin, qui étaient sollicités par de nombreuses demandes d'expérimentations en recherche appliquée ;

- trois personnes avaient successivement persévéré dans une représentation mentale erronée du fonctionnement des obturateurs et du rôle d'une clef associée au tiroir de commande des obturateurs secondaires, malgré des alarmes visuelles et sonores, jusqu'à ce qu'une quatrième personne, l'agent responsable de la « gestion » des obturateurs, leur fasse prendre conscience de la situation dangereuse dans laquelle ils opéraient. La formation des opérateurs sur les systèmes d'obturation,

298. Le faisceau de neutrons peut, après un obturateur de tête, être dirigé vers plusieurs canaux, chacun d'eux étant équipé de son propre obturateur, dit secondaire.

299. À volet, à barillet, à bouchon liquide, etc.

300. Il s'agissait d'obturateurs dits à double action, dont la membrane était maintenue en position fermée par de l'air sous pression de chaque côté de celle-ci. Ils ont été remplacés par des obturateurs à ressort et à air comprimé, de sorte qu'en cas de baisse de la pression de l'air d'un côté de la membrane, celle-ci soit maintenue en position d'obturation par le ressort agissant de l'autre côté. 
leurs conditions de fonctionnement, les balises et les alarmes associées (visuelles et sonores) apparaissait donc insuffisante ;

- l'exercice des missions incombant à la Commission interne de sécurité (CIS) était alors réduit pour ce qui concerne les dispositifs expérimentaux et les expériences.

Dans le cas du réacteur ORPHEE, la concertation entre l'exploitant de ce réacteur et le Laboratoire Léon Brillouin (LLB $\left.{ }^{301}\right)$, de qui dépendaient les expérimentateurs, était apparue perfectible, ce qui ne permettait pas au chef d'installation d'exercer clairement ses responsabilités en matière de sûreté pour ce qui concernait les dispositifs expérimentaux.

Les deux événements ont conduit à étendre formellement les responsabilités en matière de sécurité des personnes et des biens des deux chefs d'installation concernés aux dispositifs expérimentaux compris, à l'intérieur de leurs installations nucléaires de base, et à renforcer la formation des opérateurs chargés des dispositifs expérimentaux.

Ainsi, dans le cas du réacteur ORPHEE, une convention, signée en septembre 1989 entre le chef d'installation et le LLB, a précisé les interfaces entre les installations du réacteur et les dispositifs expérimentaux, et prévu la désignation d'un chef d'installation et d'un ingénieur de sécurité pour les activités expérimentales. Elle a fixé les responsabilités respectives de ces derniers et leurs rapports avec le chef de l'installation ORPHEE. Le chef d'installation des activités expérimentales a eu la responsabilité de la sécurité des personnes et des biens dans le cadre des règles générales d'exploitation du réacteur, et a été chargé :

- de la formation des opérateurs impliqués dans les activités expérimentales,

- de l'établissement des consignes correspondantes et de leur bonne application,

- d'un examen de toutes les modifications notables des moyens expérimentaux existants ou de la mise en œuvre de moyens nouveaux,

l'autorisation d'exploitation des dispositifs expérimentaux (ou de modifications jugées importantes de dispositifs déjà autorisés) étant conditionnée, comme pour le RHF, à un examen par une Commission interne de sécurité.

\subsection{Les dispositions de renforcement de la sûreté des réacteurs de recherche français après l'accident de Fukushima Daiichi}

L'accident survenu à la centrale nucléaire de Fukushima Daiichi le 11 mars 2011 a conduit le Premier ministre français à demander au président de l'ASN, le 23 mars 2011, de réaliser une étude de la sûreté des installations nucléaires, en priorité les réacteurs électronucléaires, au regard de cet accident. Cette étude devait porter sur cinq points : les risques d'inondation, de séisme, de perte des alimentations électriques et de perte

301. Le LLB est une unité mixte de recherche (UMR) associant le CEA et le CNRS. 
du refroidissement, ainsi que la gestion opérationnelle des situations accidentelles. Le Premier ministre souhaitait que soit examiné, installation par installation, si des améliorations en matière de sûreté étaient nécessaires et cela en cohérence avec les travaux menés dans le cadre européen par le groupe ENSREG et l'association WENRA. Il souhaitait que les premières conclusions de ces travaux soient présentées à la fin 2011.

Dans le même temps, le Conseil européen a demandé à l'ensemble des pays de l'Union européenne, lors de sa réunion des 24 et 25 mars 2011, la réalisation de tests de résistance (stress tests) de leurs réacteurs électronucléaires, à l'égard d'éventuelles agressions naturelles (séisme et inondation principalement) d'une ampleur dépassant les caractéristiques retenues pour la conception des installations (agressions dites extrêmes).

Pour répondre à la demande du Premier ministre, l'Autorité de sûreté nucléaire a demandé aux exploitants d'installations nucléaires EDF, CEA, AREVA et l'Institut LaueLangevin, par douze décisions en date du 5 mai 2011, de présenter :

- « les dispositions prises lors du dimensionnement de l'installation et la conformité de celle-ci aux exigences de conception qui lui sont applicables ${ }^{302}$;

- la robustesse de l'installation au-delà de ce pour quoi elle est dimensionnée, en identifiant en particulier, d'une part les situations qui conduiraient à une brusque dégradation de l'accident (" effet falaise »), d'autre part les mesures permettant d'éviter ces situations ;

- des propositions de renforcement du niveau de sûreté de l'installation et de l'organisation en cas de crise ".

Les études menées par les exploitants ont constitué les « évaluations complémentaires de sûreté » (ECS).

Comme cela a été expliqué au paragraphe 7.3.2, la conception des réacteurs de recherche français leur permet, en règle générale ${ }^{303}$, d'atteindre rapidement après leur arrêt un état ne nécessitant pas de circulation d'eau forcée et peu de moyens d'alimentation électrique (pour la surveillance de l'installation, des rejets éventuels...). De plus, leurs inventaires radiologiques sont bien entendu significativement inférieurs à ceux qui existent dans les réacteurs du parc électronucléaire. En revanche, il convient de tenir compte des risques spécifiques qu'ils présentent (construction dans des zones de sismicité notable ou en aval de barrages, à proximité de zones urbaines...).

C'est pourquoi des ECS ont été menées pour les réacteurs de recherche, selon le cahier des charges défini par l'ASN, qui reprenait celui qui avait été proposé par l'association WENRA pour les stress tests des réacteurs électronucléaires européens, augmenté d'un volet concernant les prestataires des exploitants. Des priorités ont

302. Il s'agissait ici de faire un point complet sur ces sujets, intégrant les éléments et justifications contenus dans les rapports de sûreté (dernières versions en vigueur), les écarts ou anomalies détectés au fil du temps et leur traitement, les réexamens de sûreté passés.

303. Hormis dans le cas du réacteur Jules Horowitz. 
toutefois été établies en fonction des risques associés à ces réacteurs (selon une approche "proportionnée »).

Concernant les prestataires, l'accident de Fukushima Daiichi a en effet montré que la capacité d'un exploitant et le cas échéant de ses prestataires à s'organiser pour travailler en condition d'accident grave était un élément essentiel de la maîtrise de telles situations. Cette capacité à s'organiser est également un élément essentiel de la prévention de tels accidents, de la maintenance des installations et de la qualité de leur exploitation. Ainsi, les conditions de recours à la sous-traitance revêtent une importance particulière et doivent permettre à l'exploitant de conserver l'entière maîtrise et l'entière responsabilité de la sûreté de son installation. Il est apparu nécessaire que cet aspect soit abordé dans les évaluations complémentaires de sûreté (les sujets qui devaient être traités par les exploitants sont indiqués dans le focus à la fin du présent chapitre).

Les ECS ont consisté à évaluer le comportement des réacteurs de recherche pour des agressions extrêmes, essentiellement séismes et inondations, ainsi qu'en cas de perte totale des alimentations électriques ou de la source froide externe ${ }^{304}$, et à la gestion d'accidents graves, cela dans un contexte où tout ou partie des installations d'un site pourrait être affecté de façon durable.

Il convient de souligner que l'ensemble des ECS menées en France, que ce soit pour les réacteurs électronucléaires ou pour les réacteurs de recherche (ou pour d'autres types d'INB), sont à placer dans le contexte général de la recherche d'améliorations de leur sûreté, qui s'appuie sur :

- la prise en compte du retour d'expérience,

- des réexamens de sûreté décennaux qui comportent une réévaluation des règles et des exigences de sûreté en fonction des avancées des connaissances.

De façon générale, bien qu'ils conclurent à un dimensionnement suffisant de leurs installations pour faire face à des agressions naturelles plausibles, les exploitants de réacteurs de recherche ont été amenés à proposer la mise en place de dispositions complémentaires, principalement pour augmenter l'autonomie des moyens prévus sur les sites correspondants pour faire face à des situations durables pouvant affecter le refroidissement à long terme du réacteur ${ }^{305}$ - pouvant découler d'une perte d'alimentations électriques - ou pour renforcer les dispositions de gestion d'une crise de grande ampleur.

Plus précisément, les exploitants des réacteurs de recherche français ont évalué les marges disponibles, au-delà des sollicitations sismiques de référence ${ }^{306}$, pour des " équipements essentiels » (parfois dénommés SSC-clés) en termes de sûreté, comme les bâtiments et les piscines des réacteurs, les clapets de convection naturelle, les vannes

304. L'eau provenant de l'extérieur de l'installation et pouvant être utilisée pour l'évacuation de la chaleur dégagée par le réacteur.

305. Par exemple, sans moyens de refroidissement particulier, l'évaporation progressive de l'eau d'une piscine de réacteur conduirait à devoir effectuer des apports d'eau pour éviter le dénoyage du cœur.

306. Sollicitations retenues lors des plus récentes réévaluations sismiques. 
de sécurité des canaux neutroniques... Le comportement d'équipements susceptibles de constituer des agresseurs potentiels de ces « équipements essentiels » a également été examiné (les ponts de manutention en particulier).

L'expertise des ECS transmises à l'Autorité de sûreté nucléaire par les exploitants des réacteurs de recherche français a été réalisée par l'IRSN ; l'ASN a également sollicité les avis du groupe permanent d'experts pour les réacteurs nucléaires.

Les marges estimées par les exploitants des réacteurs de recherche ont conduit l'ASN à considérer que les installations pouvaient poursuivre leur fonctionnement, mais que des améliorations devaient être mises en œuvre afin de disposer d'un « noyau dur » (voir le focus ci-après) d'équipements particulièrement robustes pour faire face à des agressions extrêmes telles qu'un séisme (" séisme noyau dur » [SND] et ses effets induits). La mise en œuvre de ces « noyaux durs » a été formellement prescrite en 2013 aux exploitants des réacteurs de recherche par un certain nombre de décisions de l'ASN. Ces « noyaux durs » doivent viser :

- " à prévenir un accident grave ou en limiter la progression ;

- à limiter les rejets radioactifs massifs ;

- à permettre à l'exploitant d'assurer les missions qui lui incombent dans la gestion d'une crise " ;

par des renforcements appropriés de la défense en profondeur.

Concernant le dernier point évoqué ci-dessus, il est en particulier apparu nécessaire, de façon générale pour les réacteurs de recherche, que les dispositions de surveillance post-accidentelle soient renforcées en matière de diagnostic de l'état de ces réacteurs en situations extrêmes, notamment pour disposer d'une information sur la position des clapets de convection naturelle ou des éléments absorbants de sécurité.

Dans ses décisions de 2013, l'Autorité de sûreté nucléaire a formulé des prescriptions concernant les situations à prendre en compte pour la conception des «noyaux durs » et particulièrement le séisme « noyau dur » (SND) :

- Les situations (dites « situations noyau dur ») à prendre en compte sont :

- la perte totale des alimentations électriques n'appartenant pas au « noyau dur »;

- la perte totale de la source froide (de refroidissement du réacteur) n'appartenant pas au « noyau dur »;

- les agressions externes retenues pour le « noyau dur »;

- les situations résultant de l'état de l'installation, du site et de son environnement après une ou des agressions externes retenues pour le « noyau dur »,

- Le SND doit :

- être enveloppe du séisme majoré de sécurité (SMS) du site, majoré de $50 \%$;

- être enveloppe des spectres définis de manière probabiliste avec une période de retour de l'ordre de 20000 ans ; 
- prendre en compte pour sa définition les effets de site particuliers et notamment la nature des sols.

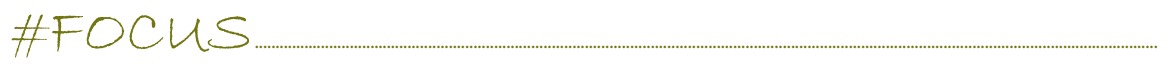

\section{Le concept de « noyau dur»}

Le renforcement de la sûreté d'installations telles que les réacteurs nucléaires, à la lumière des enseignements tirés de l'accident survenu au mois de mars 2011 à la centrale nucléaire de Fukushima Daiichi, a eu pour objectif de limiter autant que possible les rejets radioactifs à court, moyen et long termes en cas de survenue d'une agression externe d'une ampleur extrême, avec perte durable de sources (sources électriques, source froide d'un site). Il est en effet apparu souhaitable que, dans de telles situations, une installation nucléaire n'accroisse pas les difficultés auxquelles pourraient déjà devoir faire face les équipes de secours aux populations. En cas de catastrophe naturelle du type de celle survenue au Japon en mars 2011, l'environnement du site affecté serait très fortement dégradé, rendant difficile la mise en œuvre d'actions de protection des populations présentes dans le voisinage du site ainsi que, à plus long terme, la gestion des territoires contaminés.

Ainsi, le « noyau dur » post-Fukushima comprend des dispositions permettant d'assurer l'ensemble des fonctions fondamentales de sûreté, dans les situations évoquées précédemment (« situations noyau dur »).

Par ailleurs, il est apparu nécessaire que le " noyau dur » soit défini en considérant la perte de l'ensemble des dispositions déjà mises en œuvre au titre de la défense en profondeur, dont la robustesse à des agressions naturelles d'intensités significativement supérieures à celles retenues pour le dimensionnement de l'installation ne peut généralement pas être établie de façon certaine.

Ainsi, le « noyau dur " inclut des dispositions suffisamment robustes pour prévenir, autant que possible, la fusion de combustible (en cœur ou en piscine d'entreposage) ${ }^{307}$ dans les situations évoquées précédemment, ce qui comprend l'arrêt de la réaction nucléaire en chaîne et le maintien du refroidissement du combustible, ainsi que des dispositions visant à limiter les rejets, de façon à réduire les conséquences radiologiques en cas de fusion de combustible, en termes d'étendue et de durée. En complément, les missions qui incombent à l'exploitant en situation de crise doivent pouvoir être assurées. Pour cela, des dispositions du « noyau dur » doivent permettre l'accès des équipes de crise aux informations indispensables à l'appréciation de l'état des installations et à la préparation des interventions sur le site. En cas de rejets radioactifs dans l'environnement, l'exploitant doit par ailleurs être en capacité d'évaluer les conséquences de ces rejets à partir non seulement des données disponibles dans les installations, mais également de mesures réalisées dans l'environnement (mesures météorologiques, mesures de débit de dose et d'activité

307. Principe repris dans les différentes décisions de l'ASN formulées en 2013 aux exploitants. 
radiologique). Ces informations doivent permettre à l'exploitant et aux pouvoirs publics de prendre les décisions qui leur incombent respectivement pour assurer la protection des personnes présentes sur le site et des personnes du public. À cet égard, il est dès lors indispensable que le site dispose de moyens de communication opérationnels avec l'extérieur dans les situations considérées.

Pour la définition du « noyau dur », une attention doit également être portée aux systèmes dits « systèmes supports » qui permettent le fonctionnement des systèmes assurant directement les fonctions de sûreté. Il s'agit notamment des systèmes de production et de distribution électrique (groupes électrogènes ou batteries, tableaux électriques), de contrôle-commande ou de ventilation (qui assurent le conditionnement thermique des locaux). Pour ces systèmes, une indépendance et une diversification par rapport aux moyens existants sont recherchés.

Pour les installations nucléaires existantes ou dont le projet ou la construction sont déjà bien avancés (EPR Flamanville 3, réacteur Jules Horowitz), le « noyau dur » sera en définitive constitué de structures, systèmes et composants (SSC) existants, renforcés si besoin afin qu'ils soient opérationnels en cas d'agression extrême, et de SSC nouveaux.

La définition des « niveaux » des agressions extrêmes à retenir pour dimensionner le « noyau dur » n'est pas aisée, notamment en matière d'aléa sismique. Les approches classiques utilisées pour l'évaluation de l'aléa sismique ont été complétées en utilisant des méthodes probabilistes, pour viser notamment des séismes associés à une période de retour de 20000 ans tel que demandé par l'ASN dans ses prescriptions aux exploitants.

Les rapports des évaluations complémentaires de sûreté menées par les exploitants, établis selon le plan indiqué dans le focus plus loin et intégrant les dispositions complémentaires proposées (« noyaux durs » en particulier), ont été rendus publics ${ }^{308}$. Il est à noter que ces évaluations ont dans certains cas mis en évidence des nonconformités (matérielles, d'études...) relatives à des « éléments essentiels », qui devaient donc être traitées.

L'évaluation complémentaire de sûreté du $\mathrm{RHF}^{309}$ est plus particulièrement développée ci-après, compte tenu des spécificités de cette installation en termes de risques sismiques ou d'inondation, ou encore de proximité urbaine. Cette évaluation complémentaire de sûreté a conduit par exemple à la mise en place (échelonnée de 2012 à 2016) dans cette installation de différents moyens conçus et dimensionnés notamment au SND (voir les figures 10.8 et 10.9) :

- un système d'arrêt d'urgence sismique (ARS) ;

308. Sur le site internet de l'ASN.

309. Rapport RHF n 399 sur le site internet de l'ASN. 


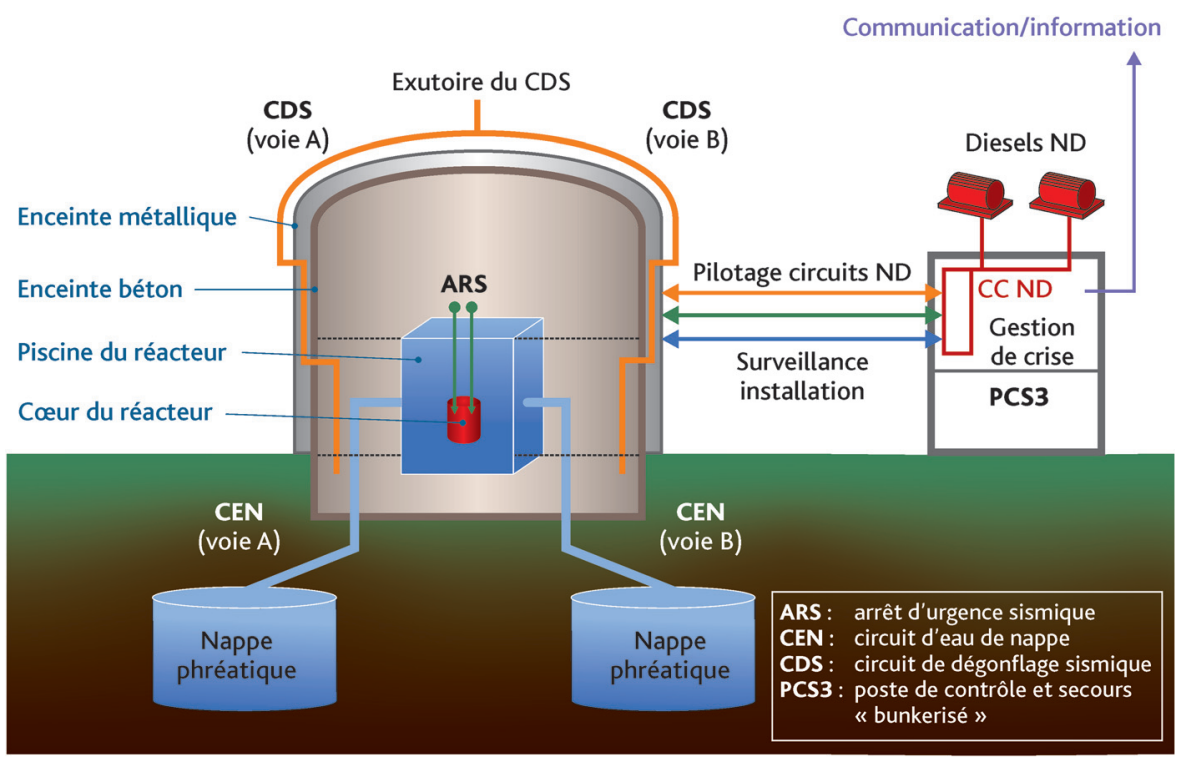

Figure 10.8. Schéma de principe des dispositions complémentaires mises en place pour le RHF après l'accident de Fukushima Daiichi. (c) Georges Goué/IRSN.

- un circuit de renoyage ultime (CRU) permettant la mise en communication du bloc-pile, de faible volume $\left(15 \mathrm{~m}^{3}\right)$, avec la piscine du réacteur de grand volume $\left(500 \mathrm{~m}^{3}\right)$;

- un circuit de pompage d'eau dans la nappe phréatique (CEN), permettant de pallier un éventuel dénoyage du cœur du réacteur ; son débit est de $250 \mathrm{~m}^{3} / \mathrm{h}$ (pour chacune des deux voies), alors que le circuit de refroidissement d'eau de secours (CES) installé en 2006 a une capacité de seulement $60 \mathrm{~m}^{3} / \mathrm{h}$;

- une nouvelle ventilation de sauvegarde du bâtiment du réacteur (circuit de dégonflage sismique [CDS]) ;

- un nouveau poste de gestion de crise (bâtiment $\mathrm{PCS}^{310} 3$ ) ;

- des dispositifs spécifiques (au PCS 3) de surveillance de quelques paramètres-clefs de l'état de l'installation (flux neutronique dans le cœur - deux nouvelles chambres de mesure neutronique ont été accrochées au bloc-pile dans la piscine en eau légère -, configuration des clapets de convection naturelle, niveaux d'eau dans le bloc-pile et dans la piscine, mesure de pression dans le hall du réacteur et dans l'espace annulaire situé entre les deux enceintes) - dont les valeurs seraient également transmises automatiquement au centre de crise de l'IRSN ${ }^{311}$ en cas d'accident.

310. Poste de contrôle et secours.

311. Pour conforter le diagnostic de l'installation avec l'exploitant (dans le cadre de la mise en œuvre de la démarche 3D-3P évoquée au paragraphe 7.7). 

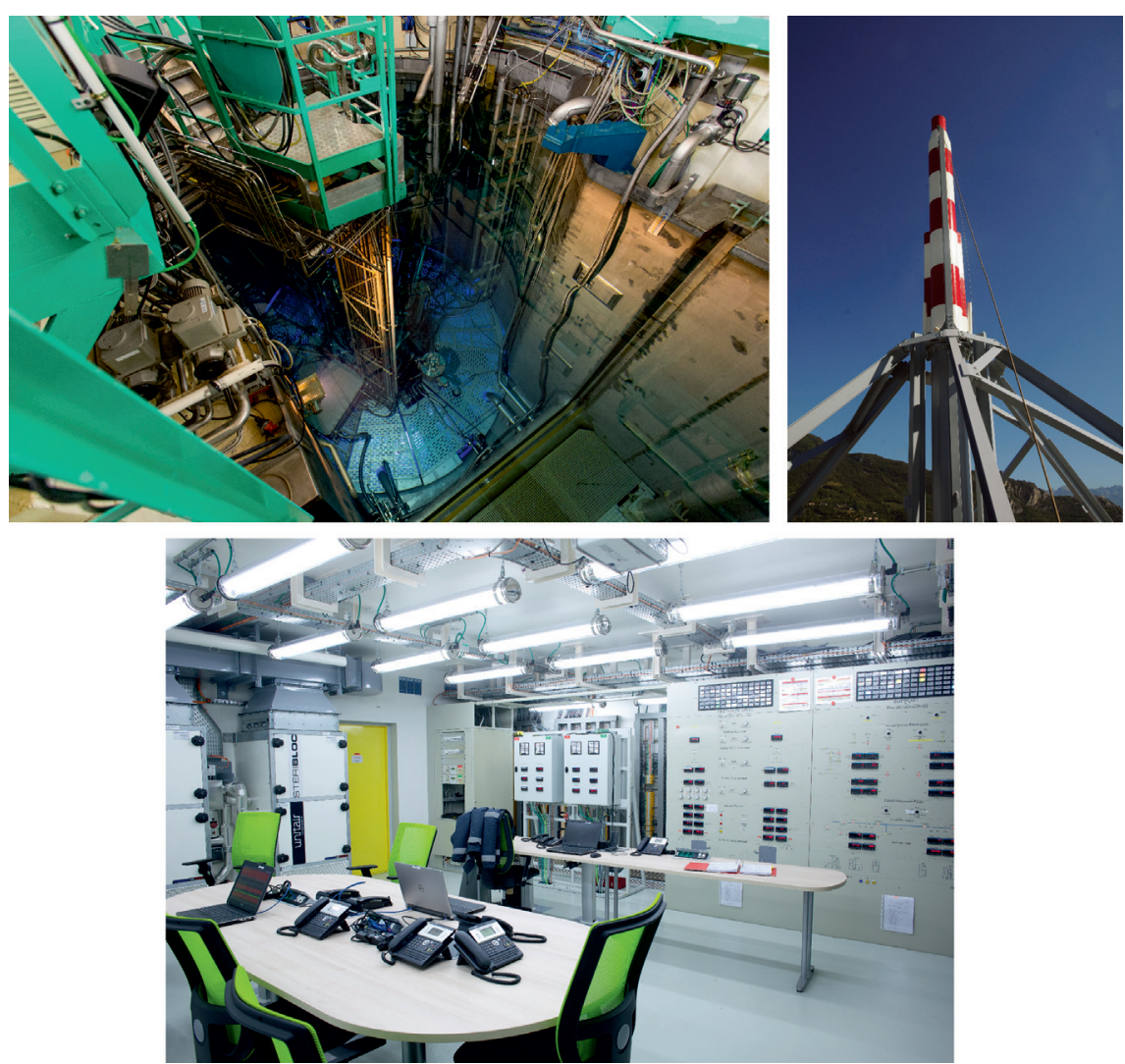

Figure 10.9. Trois dispositions retenues pour le RHF après l'accident de Fukushima Daiichi, toutes conçues et dimensionnées au séisme et à l'inondation extrêmes : en haut à gauche, apparaît notamment le coude d'arrivée dans la piscine du circuit CEN. @ IRSN, en haut à droite, la cheminée du CDS située sur le dôme de l'enceinte métallique. @ ILL, en bas, la salle de gestion de crise au PCS 3. @ Jean-Marie Huron/ Signatures/IRSN.

Ces systèmes, en grande majorité redondants ${ }^{312}$ et automatiques, sont alimentés électriquement par des groupes électrogènes de sauvegarde, implantés dans le nouveau bâtiment PCS 3 qui a été conçu, non seulement pour résister aux agressions extrêmes considérées pour le site du RHF (SND, équivalent à deux fois le SMS dans les fréquences d'intérêt), mais aussi à la rupture de barrages situés en amont (le PCS 3 est surélevé à une hauteur de six mètres au-dessus de la plateforme du site du RHF). Ils sont pleinement opérationnels depuis 2016.

Concernant l'arrêt d'urgence sismique (ARS), il convient de mentionner que le contrôle-commande du système de protection du réacteur avait déjà été adapté pour

312. II n'y a qu'un seul PCS 3. 
que la chute des barres de sécurité ${ }^{313}$ intervienne automatiquement en cas de sollicitations sismiques, en réglant le seuil de déclenchement à $0,01 \mathrm{~g}^{314}$. Il a été entièrement refait pour le rendre indépendant des autres protections du réacteur et il a été qualifié au séisme " noyau dur ». Cette disposition assure la chute des barres de sécurité même en cas de séisme sans phase de faibles mouvements (ondes de compression dites ondes P) avant la phase des mouvements forts (ondes de cisaillement dites ondes S).

Toujours pour le RHF, l'exploitant a étudié deux situations de rupture de barrage(s) situé(s) en amont sur le Drac et leur impact possible sur les « équipements essentiels »; il s'agit :

- de la rupture du barrage de Monteynard, celle-ci pouvant entraîner la rupture du barrage de Notre-Dame-de-Commiers situé en aval ;

- de la rupture de tous les barrages situés sur le Drac, qui conduit à la cote la plus élevée physiquement possible à Grenoble.

Ces études ont conduit l'exploitant à prendre en compte une hauteur hypothétique de six mètres d'eau sur la plateforme du RHF. La conception du nouveau bâtiment de gestion de crise (PCS 3) en a tenu compte. Les grandes ouvertures du bâtiment du réacteur (portes-camions...) ont été renforcées de telle sorte qu'elles puissent résister au SND et à une hauteur d'eau de six mètres sur le site en conservant une étanchéité suffisante de façon à éviter des entrées massives d'eau dans ce bâtiment.

Pour la définition d'un séisme « noyau dur » (SND), la question des effets de site se pose particulièrement pour le RHF, ce réacteur étant implanté dans une vallée alluvionnaire (la hauteur des alluvions au-dessus du rocher est d'environ $700 \mathrm{~m}$; elles sont composées en partie d'argile). C'est pourquoi l'exploitant s'est impliqué dès les années 2000 dans des travaux d'études et recherches sur les effets de site (projet CASHIMA et, plus récemment, projet SIGMA ${ }^{315}$ ) et a fait réaliser des mesures (de type cross hole ${ }^{316}$ ) ainsi que des simulations numériques à deux dimensions en vue de mieux apprécier ces effets de site. Les coefficients multiplicatifs appliqués aux spectres - préalablement établis sans prendre en compte les effets de site - sont compris entre 1,3 (pour les fréquences supérieures à $3 \mathrm{~Hz}$ ) et 2 (pour les fréquences inférieures à $0,3 \mathrm{~Hz}$ ). L'accélération maximale du sol à fréquence infinie (PGA) du SND est de 0,6 g, à comparer à une valeur voisine de $0,3 \mathrm{~g}$ retenue lors de la réévaluation sismique du début des années

313. Il s'agit de cinq barres absorbantes situées dans la zone du réflecteur, en dehors de l'élément combustible constituant le cœur du réacteur dans lequel coulisse la barre de pilotage.

314. La valeur du seuil de déclenchement d'un arrêt d'urgence est choisie inférieure au PGA, afin de disposer d'un temps suffisant pour la mise en œuvre de cet arrêt d'urgence. Une valeur de 0,01 g est typiquement atteinte lors de l'arrivée des ondes sismiques primaires (ondes $P$ ) ; des accélérations du niveau des PGA (quelques dixièmes de g) sont détectées plusieurs secondes après, au moment de l'arrivée des ondes $\mathrm{S}$, qui sont les plus dommageables pour les équipements.

315. Voir l'ouvrage «État des recherches dans le domaine de la sûreté des réacteurs à eau sous pression », Collection sciences et techniques, IRSN/EDP Sciences, 2017. De nombreux partenaires européens, industriels et organismes de recherche, y compris des universités, sont impliqués dans ces deux projets.

316. Mesures de la vitesse des ondes de cisaillement à $30 \mathrm{~m}$ de profondeur - voir le même ouvrage. 
2000. Finalement, dans les fréquences d'intérêt pour les structures, systèmes et composants, le SND pour le RHF est deux fois supérieur au SMS issu de la réévaluation sismique effectuée au début des années 2000.

Les « équipements essentiels » ont fait l'objet d'une vérification de leur tenue sismique au SND (ponts et portiques de manutention, vannes de sécurité des canaux neutroniques...), y compris par des essais réalisés sur table vibrante. Cela a conduit l'exploitant à réaliser d'ores et déjà des renforcements :

- du portique de manutention des hottes à combustible,

- de gros équipements proches de la margelle de la piscine (« source froide » verticale...),

- du poste d'entretien des hottes (agresseur potentiel de l'enceinte de confinement),

- des grandes ouvertures dans le bâtiment du réacteur (porte à camion...).

L'exploitant du RHF a été amené à apporter quelques évolutions au « noyau dur » tel qu'initialement prévu. Ces évolutions ont concerné en particulier le tracé d'implantation du système de ventilation ultime, le cheminement des câbles pour qu'ils ne soient pas emportés en cas d'inondation extrême (ils sont enterrés à 5 mètres de profondeur), la prise en compte des risques chimiques liés à l'environnement du site ; le PCS 3 est ainsi conçu pour être habitable et opérationnel en cas d'accident impliquant un rejet de phosgène en provenance de la plateforme chimique de Pont de Chaix (système de ventilation/filtration adapté).

Comme cela a été indiqué précédemment, le principe de «bloc-eau » a été adopté pour la conception des réacteurs du CEA tels qu'OSIRIS, ORPHEE ou le réacteur Jules Horowitz, ce qui constitue une option de conception favorable pour le refroidissement du réacteur, en assurant le maintien dans le réacteur d'un volume d'eau suffisant en cas de fuite, hors du bloc-pile, d'une portion de tuyauterie du circuit de refroidissement du cœur. Toutefois, il n'est pas possible d'exclure totalement, en situations extrêmes, une perte d'étanchéité de locaux ou casemates dans lesquels sont situées ces portions de tuyauteries, même si des marges existent dans le dimensionnement de ces locaux ou casemates $^{317}$.

Pour les réacteurs en exploitation (ORPHEE, CABRI...), différentes dispositions complémentaires ont été proposées par le CEA : groupes électrogènes supplémentaires, dispositifs d'appoint alimentés en eau par des moyens mobiles, moyens de mesure complémentaires (niveaux d'eau...), panneaux de repli permettant une surveillance des installations après une agression extrême. Par ailleurs, des dispositions renforcées ont été programmées entre 2015 et 2018 pour permettre une gestion de crise efficace même en situation extrême (mises en place d'équipes de reconnaissance des installations après une agression extrême, renforcement ou création de locaux abritant des équipements et systèmes qui pourraient être utilisés en situation extrême).

317. Dimensionnés au SMS. 
Pour ce qui concerne le réacteur Jules Horowitz, l'évaluation complémentaire de sûreté ${ }^{318}$ a été menée par le CEA sur la base de l'état de la conception de ce réacteur en 2011. Des dispositions de diverses natures ont été retenues pour la poursuite du projet, telles que :

- l'introduction de marges de dimensionnement supplémentaires pour certains équipements (ancrages des racks d'entreposage du combustible neuf, galets et voies de roulement de la passerelle passant au-dessus de la piscine du réacteur...). En outre, des dispositifs de mesure (position des clapets de convection naturelle, niveaux d'eau dans les piscines et température de l'eau de la piscine du réacteur) ont été retenus comme équipements " noyau dur »;

- la décision de disposer, en plus des groupes électrogènes de sauvegarde et des possibilités de réalimentation électrique par un groupe mobile du centre de Cadarache, d'un groupe d'ultime secours (GUS) à demeure, qualifié en tant que « noyau dur » (installé notamment sur une plateforme non inondable) ;

- la création de deux piquages sur les circuits de réfrigération de sauvegarde (RUS), pour permettre une alimentation en eau froide par des moyens externes (camions citernes équipés d'une pompe autonome) ;

- l'entreposage d'un volume d'eau suffisant dans les capacités de vidange des piscines pour pouvoir assurer un appoint d'eau de secours dans ces piscines, avec un dimensionnement au séisme « noyau dur » (SND) du circuit permettant cet appoint ;

- l'ajout d'un dispositif d'arrêt d'urgence du réacteur en cas de détection sismique.

\section{\#FOCUS}

\section{Sujets traités dans les rapports relatifs aux évaluations complémentaires de sûreté menées par les exploitants après l'accident de la centrale nucléaire de Fukushima Daiichi}

Les rapports relatifs aux évaluations complémentaires de sûreté (ECS) ont été rédigés selon le plan générique suivant :

- caractéristiques de l'installation et son état actuel,

- identification des risques d'effet falaise et des structures et équipements essentiels :

318. Dossier rendu public sur le site de l'ASN : « Réacteur Jules Horowitz - Évaluation complémentaire de la sûreté au regard de l'accident survenu à la centrale de Fukushima I » (CEA/DEN/CAD/DIR/ CSN DO 575 13/09/11). 
L'effet falaise est défini comme une altération brutale du comportement d'une installation, que suffit à provoquer une légère modification du scénario envisagé pour un accident dont les conséquences sont alors fortement aggravées $^{319}$,

- séismes : aléas considérés (lors des études de conception, puis lors des réévaluations de sûreté), quantification des marges,

- inondations externes : aléas considérés (lors des études de conception, puis lors des réévaluations de sûreté), quantification des marges,

- autres phénomènes naturels extrêmes,

- perte des alimentations électriques et perte des systèmes de refroidissement,

- gestion des accidents graves,

- conditions de recours aux entreprises prestataires :

- champ des activités concernées avec les justifications associées,

- modalités de choix des prestataires : exigences en matière de qualification, formation à la sûreté nucléaire et à la radioprotection...,

- dispositions prises pour permettre des conditions d'intervention satisfaisantes pour les entreprises prestataires, organisation mise en œuvre pour la radioprotection des intervenants,

- modalités de surveillance des activités sous-traitées, en particulier la manière dont l'exploitant continue d'assurer sa responsabilité en matière de sûreté nucléaire et de radioprotection,

- synthèse, incluant d'éventuelles propositions de dispositions complémentaires. 